\title{
A semi-analytical model of hydroelastic slamming
}

\author{
Zhe Sun ${ }^{1}$, A. Korobkin ${ }^{2,3}$, X.P. Sui ${ }^{1}$, Zhi,Zong ${ }^{1,4,5^{*}}$ \\ ${ }^{1}$ School of Naval Architecture, Dalian University of Technology, Dalian, 116024, P. R. China \\ ${ }^{2}$ School of Mathematics, University of East Anglia, Norwich Research Park, Norwich NR47TJ, United Kingdom \\ ${ }^{3}$ Lavrentyev Institute of Hydrodynamics, pr. Lavrentyeva 15, Novosibirsk 630090, Russia \\ ${ }^{4}$ State Key Laboratory of Structural Analysis for Industrial Equipment, Dalian, 116024, P. R. China \\ ${ }^{5}$ Collaborative Innovation Center for Advanced Ship and Deep-Sea Exploration, Shanghai, 200240, P. R. China \\ First Author: Zhe Sun (zsunch@163.com), Corresponding Author: Zhi Zong (zongzhi@dlut.edu.cn)
}

\begin{abstract}
A semi-analytical model of hydroelastic slamming for two-dimensional symmetric flexible bodies is presented in this paper. The hydrodynamic loads are computed using the analytical Modified Logvinovich Model (MLM) with account for elastic deflections of the body surface. The elastic deflections are described by the modal approach. The rigid and elastic motions of the body are computed at the same time together with the wetted part of the body surface. The coupled problem of elastic impact is reduced to a system of nonlinear ordinary differential equations, which is integrated in time by the implicit Newmark method with iterations at each time step. Both free fall and constant water entry cases are considered. The developed model is validated for flexible wedge and cylindrical shell water entry problems. Good agreements with existing experimental and numerical results are achieved. It is shown that the MLM significantly improves predictions of the deflections and stresses cause by the impact compared with the Wagner model of water impact. The developed model is suitable for efficient and accurate computations of water elastic impacts.
\end{abstract}

Key words: Fluid and Structure Interaction (FSI); Hydroelasticity; Slamming; MLM; Arbitrary Symmetric Shape

\section{Introduction}

Slamming is important for structural integrity of ships and marine structures. A combination of impulsive slamming loads and structural flexibility could lead to the so-called whipping problem ${ }^{[1]}$ for ships, which poses severe threat to the safety of the ships. Impacts on water have also to be taken into consideration in design of aircraft and aerospace structures subject to water landing ${ }^{[2]}$. Predictions of both the hydrodynamic loads during slamming events and the resulting structural responses have drawn attention of many researchers.

The experiment has always been an important tool to reveal the physics behind complex physical processes. Some recent and representative experimental works on slamming of flexible structures include ones by Shams et al[3], Jalalisendi and Porfiri ${ }^{[4]}$, in which the dynamics of flexible wedge and cylindrical shell during slamming were investigated. On the other hand, the recent rapid development of computational powers makes the direct simulations based on Computational Fluid Dynamics (CFD) to be one of the most popular tools of investigating such problems. Among various CFD based numerical models ${ }^{[5-7]}$, the emerging particle method is considered to be a promising approach for violent flow simulations such as slamming due to its meshless Lagrangian nature ${ }^{[8,9]}$. On the other hand, it is known that viscous effects are negligible in slamming. Hence potential flow models and corresponding methods, such as Boundary Element 
Method ${ }^{[10,11]}$, are commonly used for slamming simulations. Both the CFD and potential flow based methods are still time consuming despite the rapid growing computational capability, which is not acceptable for the initial stage of marine structure design. This could explain why two dimensional analytical models are still widely used for preliminary evaluation of slamming loads and structural responses ${ }^{[2]}$.

The first theoretical models of water impact were proposed by von Karman ${ }^{[12]}$ and Wagner ${ }^{[13]}$ in the 1930s. In these models, the impacting structures were rigid. Von Karman ${ }^{[12]}$ calculated the slamming forces using the momentum conservation law without account for the elevation of the water surface caused by the impact. The Wagner model ${ }^{[13]}$ accounts for the water surface deformation and provides reasonable pressure distributions along the wetted surfaces of bodies. Both models over-predict the slamming loads. Wagner ${ }^{[13]}$ argued that it is better for design to overestimate slamming loads than underestimate them. The Wagner model was generalized to water impact of three-dimensional ${ }^{[14-16]}$ and elastic ${ }^{[4,17-19]}$ bodies.

Khabakhpasheva and Korobkin ${ }^{[20]}$ combined the Euler-Bernoulli beam theory and the Wagner model of water impact to investigate stresses in the elastic wedge entering water at a constant speed. They derived analytical formulae for elements of the added mass matrix of elastic modes of a uniform beam with different edge conditions and a differential equation for the size of the wetted part of the elastic wedge, which made calculations fast even with large number of modes. They also provided extensive review of previous studies of elastic impacts and suggested several simplified models of impact. Shams and Porfiri ${ }^{[21]}$ further developed the normal-mode model ${ }^{[20]}$ by deriving an "exact' solution to the boundary value problem of hydroelastic impact of flexible wedges including free-drop impact condition, which along with the application of the Gram Schmidt algorithm yields a set of ordinary differential equations in closed form that can be easily integrated. The model was validated against available experimental results in terms of strains, deflections and total forces. These approaches are suitable for treatment of non-homogeneous wedge plating with several different supports as well.

The Wagner impact model was combined with a finite-element model of elastic wedge response by Korobkin, Guéret and Malenica ${ }^{[22]}$. The added mass matrix of the beam finite elements was calculated analytically. In general, the Wagner model for elastic body impact on water well describes the time evolution of strains in the wedge plating but over-predicts them. This is caused by the fact that the Wagner model over-predicts the hydrodynamic loads acting on the entering body.

There were several attempts in the past to improve prediction of the water impact loads. Wagner ${ }^{[22]}$ suggested to use his linearized hydrodynamic model, with the wetted part of the body surface approximated by a flat disc, to determine only the flow caused by impact on the water surface. He suggested to use the non-linear Bernoulli equation for the hydrodynamic pressure with the velocity potential determined by his flat-disc approximation. The resulting pressure was highly singular and not integrable at the periphery of the contact region. Later it was suggested to consider only the positive part of this non-linear pressure. Further improvements of hydrodynamic loads were suggested by Korobkin ${ }^{[23]}$, who approximately accounted for actual shape of the entering body. This idea was inspired by the success of the so-called Generalized Wagner Model (GWM) by Zhao and Faltinsen ${ }^{[24]}$, where the body boundary conditions were imposed on the actual position of the entering body without simplifications and the free-surface conditions were linearized and imposed on the pile-up height as in the original Wagner model (OWM). The model by Korobkin ${ }^{[23]}$ was named Modified Logvinovich Model (MLM), even it is very different from the Original 
Logvinovich Model (OLM) ${ }^{[25]}$, which is a type of the Wagner model with flat-disc approximation but with another reasoning for determination of the wetted part of the body surface. Surprisingly the forces by MLM were found to be very close to those by OLM for blunt bodies, which was a reason for the name MLM. Note that the OLM does not work for shapes with large deadrise angles because the OLM significantly under-predicts the size of the wetted area for such a body. The MLM was intensively compared with experimental and computations results for rigid bodies and showed good performance in most cases. Three-dimensional MLM ${ }^{[26]}$ was applied to the free-drop of panels with double curvature. The obtained panel decelerations were in good agreement with experimental results by Wraith ${ }^{[27]}$.

Tassin et al ${ }^{[28]}$ studied aircraft ditching by the $2 \mathrm{D}+\mathrm{T}$ method. In this method, a vertical control plane, which is perpendicular to the aircraft motion, was introduced. The flow caused by the ditching was approximated as two-dimensional in this plane. In this way, the original three-dimensional problem for an elongated body was reduced to a set of two-dimensional water impact problems with a body which changes its shape in time. Note that this change of shape is known in advance if the body motions are given. The aircraft fuselage was described as a one-dimensional elastic structure but each section of the aircraft was rigid. The sectional forces were calculated by the MLM which was developed further by including time-variation of the body shape during the impact on water.

In the present paper, the MLM by Tassin et al ${ }^{[28]}$ is generalized to elastic structures, where the structural response depends on the hydrodynamic loads which, in turn, depend on deflection of the structure. The hydrodynamic loads are non-linear with respect to the body deflection which makes the hydroelastic models with MLM more complicated than those with the linearized OWM. Nevertheless, the MLM can be directly coupled with a structural model without using an interface because the hydrodynamic pressure by the MLM is nonlinear with respect to the body deflection and its local speed but linear with respect to the local acceleration of the body surface.

This is the first study of elastic slamming, where the MLM of hydrodynamic loads is combined with a structural model of impacting body. To make the very first step in this direction, we do not use the advantages of MLM in full but employ the MLM only to replace a hydrodynamic CFD solver in a standard architecture CFD + Structure Solver(e.g. Modal Superposition, FEM)+Twoway Coupling ${ }^{[8,29]}$. The structural dynamics and the MLM hydrodynamics are coupled in a partitioned way, in which a standard iteration method is used to achieve high coupling accuracy.

The paper is organized as follows. Section 2 provides the equations and numerical algorithms for both the fluid and structural solvers. The obtained results for a symmetric wedges entering water at constant speed and in free drop, and cylindrical shells in free drop are discussed and compared with existing experimental or numerical results in Section 3. The conclusions are drawn in Section 4.

\section{Methodology}

In this section, equations of structural models for impacting body response and the hydrodynamic model of the flow and loads caused by the impact are presented together with details of their numerical implementations and coupling. 


\subsection{Governing equations of structural dynamics}

A flexible symmetric two-dimensional body is described by using the Cartesian coordinate system, as shown in Fig. 1. This coordinate system $y-o-z$ is used for computations of the impact hydrodynamic forces and the corresponding structure responses. The $o-y$ axis is along the initial flat and horizontal position of the water free surface, and $o-z$ axis is directed upwards along the symmetry line. Initially, $t=0$, the body touches the water surface at the origin, $y=0, z=0$, and then starts instantly to penetrate the water. The projection of the wetted body surface on the $o-y$ axis corresponds to the interval $-c(t)<y<c(t)$, where $c(t)$ should be determined as part of the solution. The position of the body surface at time $t>0$ is described by the equation $z=f(y, t)$, where

$$
f(y, t)=f_{0}(y)+f_{e}(y, t)-\zeta(t),
$$

$f_{0}(y)$ is the initial undeformed shape of the body, $f_{e}(y, t)$ is the elastic deflection of the body surface in the $z$-direction, and $\zeta(t)$ is the penetration depth of the body, see Fig. 1 . The symmetry of the body means that $f(y, t)=f(-y, t), f_{0}(y)=f_{0}(-y)$, and $f_{e}(y, t)=f_{e}(-y, t)$. In order to simplify the pressure integration along the body surface later, both the elastic, $f_{e}(y, t)$, and the rigid, $f_{0}(y)$, terms in (2.1) are combined together as $f_{d}(y, t)$ :

$$
f_{d}(y, t)=f_{0}(y)+f_{e}(y, t) \text {. }
$$

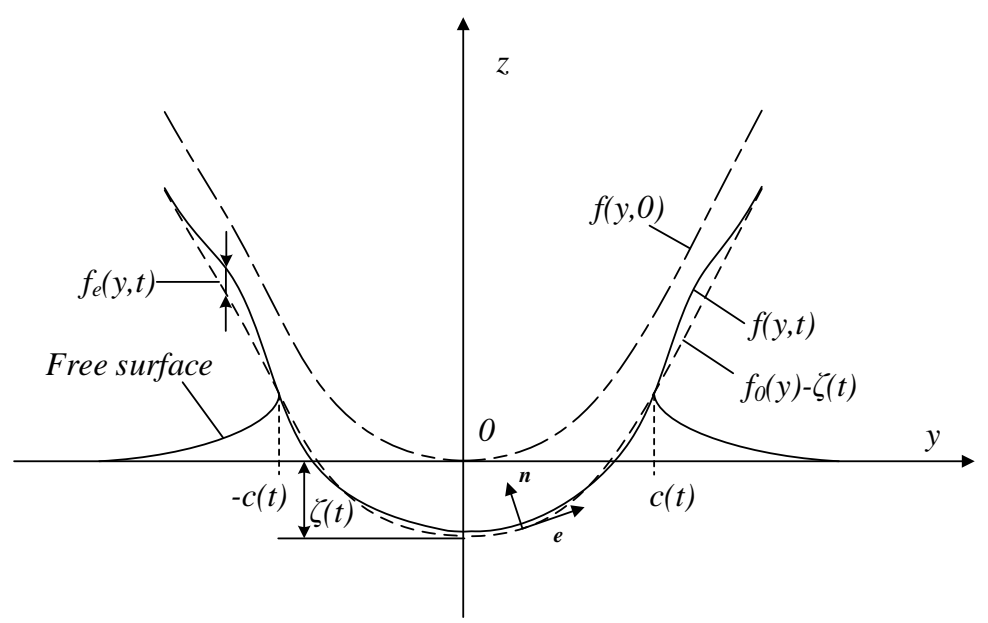

Fig.1. The coordinate system and sketch of the water entry problem for a symmetric elastic body.

The unit vectors $\boldsymbol{n}$ and $\boldsymbol{e}$, see Fig.1, represent the normal and tangential directions on the current body surface. The flexible deformation $f_{e}(y, t)$ is represented by modal superposition approach in this study with general coordinates $\mathbf{q}(t)=\left[q_{1}(t), q_{2}(t), q_{3}(t), \ldots\right]^{T}$. The following Lagrangian equations, 


$$
\left\{\begin{array}{l}
\frac{d}{d t}\left(\frac{\partial T}{\partial \dot{\zeta}}\right)+\frac{\partial U}{\partial \zeta}-\frac{\partial T}{\partial \zeta}=Q_{\zeta} \\
\frac{d}{d t}\left(\frac{\partial T}{\partial \dot{q}_{k}}\right)+\frac{\partial U}{\partial q_{k}}-\frac{\partial T}{\partial q_{k}}=Q_{q_{k}} \quad k=1,2,3, \ldots
\end{array}\right.
$$

are used to derive differential equations in time for the penetration depth $\zeta(t)$ and the general coordinates $q_{j}(t)$, where $T$ and $U$ in (2.3) are the kinetic and potential energies of the structure, $Q_{\zeta}$ and $Q_{q_{k}}$ are the non-conservative forces for the vertical rigid-body motion and the flexible modes, respectively. Overdot in (2.3) stands for time derivative. The forces $Q_{\zeta}$ and $Q_{q_{k}}$ for the structures considered in this study will be specified below.

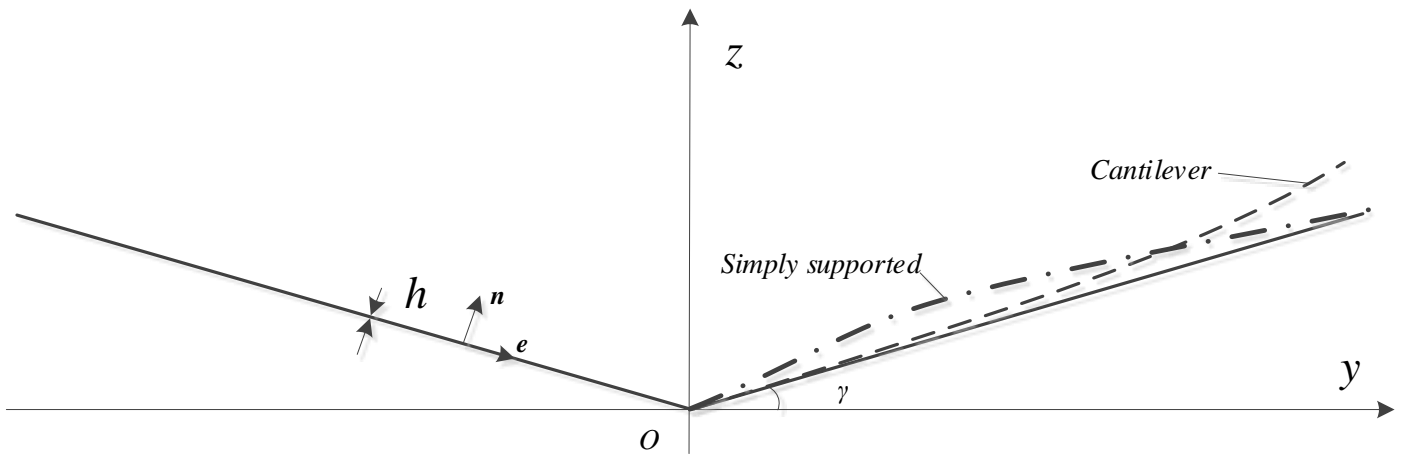

(a)

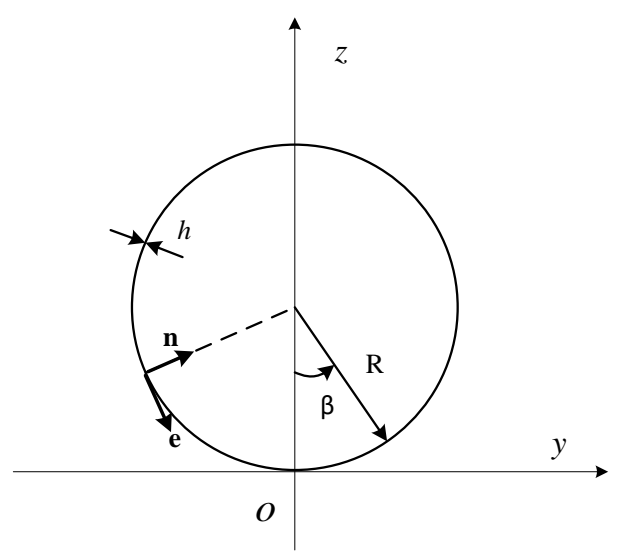

(b)

Fig.2 The sketch of the water impact problems for flexible wedge (a) and cylindrical shell (b)

Two structures are investigated in this paper: flexible wedge and cylindrical shell, which are shown in Fig.2 (a) and (b) respectively. The corresponding structural kinetic and potential energies can be found in ${ }^{[7,8]}$. The corresponding values of the parameters used in the simulations for wedges and a cylindrical shell are given in Table 1 and Table 2. 
Table 1. Parameters of elastic wedge

\begin{tabular}{|c|c|c|c|c|c|c|}
\hline & \multicolumn{3}{|c|}{ Free fall } & \multicolumn{2}{|c|}{ Free fall } & Constant entry \\
\hline Length of each beam L (m) & \multicolumn{3}{|c|}{0.13} & \multicolumn{2}{|c|}{0.3} & 0.5 \\
\hline Thickness $h(\mathrm{~m})$ & \multicolumn{3}{|c|}{0.0005} & \multicolumn{2}{|c|}{0.002} & 0.018 \\
\hline Total Mass $M(\mathrm{~kg})$ & \multicolumn{3}{|c|}{1.9} & \multicolumn{2}{|c|}{20} & - \\
\hline Deadrise angle $\gamma\left({ }^{\circ}\right)$ & \multicolumn{3}{|c|}{22} & \multicolumn{2}{|c|}{15} & 10 \\
\hline Young's modulus $E(\mathrm{~Pa})$ & \multicolumn{3}{|c|}{$6.89 \times 10^{10}$} & \multicolumn{2}{|c|}{$6.8 \times 10^{10}$} & $2.1 \times 10^{11}$ \\
\hline Material density $\rho_{s}\left(\mathrm{~kg} / \mathrm{m}^{3}\right)$ & \multicolumn{3}{|c|}{$2.7 \times 10^{3}$} & \multicolumn{2}{|c|}{$2.7 \times 10^{3}$} & $7.85 \times 10^{3}$ \\
\hline Boundary condition & \multicolumn{5}{|c|}{ Cantilever } & Simply supported \\
\hline Entry velocity $V(\mathrm{~m} / \mathrm{s})$ & 1.87 & 2.77 & 3.38 & 4 & 5 & 4 \\
\hline Case No. & 1 & 2 & 3 & 4 & 5 & 6 \\
\hline
\end{tabular}

Table 2. Parameters of cylindrical shell

\begin{tabular}{l|l}
\hline \multicolumn{2}{c}{ Parameters for cylindrical shell } \\
\hline Radius $\mathrm{R}(\mathrm{m})$ & 0.156 \\
\hline Thickness $h(\mathrm{~m})$ & 0.0051 \\
\hline Total Mass $M(\mathrm{~kg})$ & 39.2 \\
\hline Young's modulus $E(\mathrm{~Pa})$ & $2.06 \times 10^{11}$ \\
\hline Poisson's ratio $v$ & 0.3 \\
\hline Material density $\rho_{s}\left(\mathrm{~kg} / \mathrm{m}^{3}\right)$ & $7.848 \times 10^{3}$ \\
\hline Entry velocity $V(\mathrm{~m} / \mathrm{s})$ & 4.29 \\
\hline
\end{tabular}

The symmetric wedges are made of two identical Euler beams of constant thickness. The beams are installed symmetrically, therefore only the deflections for $y \geq 0$ need to be determined. The beams are either simply supported at both edges or are cantilever (clamped at the wedge vertex and free-free at other edges). The modal decomposition for the beams read

$$
f_{e}(y, t)=\boldsymbol{\psi}^{T}(s(y)) \cdot \mathbf{q}(t) n_{z},
$$

where $s$ is the curvilinear coordinate along the undeformed body surface starting from the lowest point of the body, $0<s<L, n_{z}$ is the $z$ component of the normal unit vector to the body surface, $\boldsymbol{\psi}(s(y))$ is the mode vector-function, and $\mathbf{q}(\mathrm{t})$ is the vector of the corresponding general coordinates,

$$
\begin{aligned}
& \psi(s)=\left[\psi_{1}(s), \psi_{2}(s), \psi_{3}(s), \ldots\right]^{T} \\
& \mathbf{q}(t)=\left[q_{1}(t), q_{2}(t), q_{3}(t), \ldots\right]^{T}
\end{aligned}
$$

Moreover, the mode functions satisfy the following orthogonal equations:

$$
\begin{aligned}
& \int_{0}^{L} \boldsymbol{\psi} \cdot \boldsymbol{\psi}^{T} \rho_{l} d s=\mathbf{I} \\
& \int_{0}^{L} \frac{d^{2} \boldsymbol{\psi}}{d s^{2}} \cdot \frac{d^{2} \boldsymbol{\psi}^{T}}{d s^{2}} \text { EIds }=\boldsymbol{\Lambda}
\end{aligned}
$$


where $\rho_{l}$ is the line density of the beam, $\mathbf{\Lambda}=\operatorname{diag}\left(\omega_{k}^{2}\right)$ is the diagonal matrix, $\omega_{k}$ is the $k^{\text {th }}$ natural frequency of the beam, $\omega_{k}<\omega_{k+1}, k \geq 1$, and $\mathbf{I}$ is a unit matrix. The product $\boldsymbol{\psi} \cdot \boldsymbol{\psi}^{T}$ is a matrix with elements $\left(\boldsymbol{\psi} \cdot \boldsymbol{\psi}^{T}\right)_{i j}=\psi_{i}(s) \psi_{j}(s)$.

The governing equations for the wedge dynamics are derived by determining the kinetic and potential energies and using (2.3), see ${ }^{[8]}$ for details,

$$
\left\{\begin{array}{l}
2 \cos (\gamma) \boldsymbol{\Psi}_{\mathbf{0}}{ }^{T} \ddot{\mathbf{q}}-M \ddot{\zeta}+M g=Q_{\zeta} \\
2 \ddot{\mathbf{q}}+2 \Lambda \mathbf{q}-2 \ddot{\zeta} \cos (\gamma) \boldsymbol{\Psi}_{\mathbf{0}}=\mathbf{Q}_{\mathbf{q}}
\end{array}\right.
$$

where $\gamma$ is the deadrise angle of the wedge. Note that the deadrise angle is not necessary small in the present study. The non-conservative forces $Q_{\zeta}, \mathbf{Q}_{\mathbf{q}}=\left[Q_{q_{1}}, Q_{q_{2}}, Q_{q_{3}}, \ldots\right]^{T}$ and the constant vector $\psi_{0}=\left[\psi_{01}, \psi_{02}, \psi_{03}, \ldots\right]^{T}$ are defined by

$$
Q_{\zeta}=\int_{-c}^{c} p d y
$$

$Q_{q_{k}}=\int_{-c}^{c} p \psi_{k} n_{z} d y, \quad k=1,2,3, \ldots$

$\psi_{0 k}=\int_{0}^{L} \psi_{k} \rho_{l} d s, \quad k=1,2,3, \ldots$

where $p$ is the hydrodynamic pressure acting on the wetted part of the body surface, and $g$ is the acceleration due to gravity.

The modal functions $\psi_{k}(s)$ depend on the edge conditions of the beam. These functions are determined up to constant factors, which are obtained from the normalization condition (2.7). Then for a cantilever and simply supported Euler-Bernoulli beams, assuming the beam material is linear, elastic and obeys Hooke's law; plane beam sections remain plane and perpendicular to the neutral axis at any time ${ }^{[30]}$, the $k^{\text {th }}$ mode functions are given by, see ${ }^{[31]}$, respectively:

$$
\begin{aligned}
& \psi_{k}(s)=\frac{1}{\sqrt{M_{b}}}\left[\cosh \left(\frac{2 \mu_{k} s}{L}\right)-\cos \left(\frac{2 \mu_{k} s}{L}\right)-\frac{\cos \left(2 \mu_{k}\right)+\cosh \left(2 \mu_{k}\right)}{\sin \left(2 \mu_{k}\right)+\sinh \left(2 \mu_{k}\right)}\left(\sinh \left(\frac{2 \mu_{k} s}{L}\right)-\sin \left(\frac{2 \mu_{k} s}{L}\right)\right)\right] \\
& \psi_{k}(s)=\sqrt{\frac{2}{M_{b}}} \sin \left(\frac{2 \mu_{k} s}{L}\right)
\end{aligned}
$$

where $M_{b}=\rho_{l} L$ is the mass of each beam. In (2.13), $\mu_{k}$ is the $k^{\text {th }}$ root of the relation $\cos \left(2 \mu_{k}\right)+\cosh \left(2 \mu_{k}\right)=-1$. For the first three modes: $\mu_{1}=0.9375, \mu_{2}=2.3470, \mu_{3}=3.9274$. In (2.14), $\mu_{k}=\frac{k \pi}{2}$, where $k=1,2,3, \ldots$ 
For a cylindrical shell of radius $\mathrm{R}$, we have

$f_{e}(y, t)=w(\beta, t) \mathrm{n}_{z}+v(\beta, t) e_{z}$,

where $w$ and $v$ are the normal and tangential displacements of the shell elements respectively, $e_{z}$ is the $z$ component of the tangential unit vector to the body surface, and $\beta$ is the central angle of a point on the shell with the horizontal coordinate $y$ (with respect to the undeformed circle), i.e. $y=R \cdot \sin (\beta)$. Similar to the case of flexible wedge, $w$ and $v$ are represented using the modal superposition approach:

$w(\beta, t)=\boldsymbol{\psi}^{T}(\beta) \mathbf{q}(t)$

$v(\beta, t)=\tilde{\boldsymbol{\psi}}^{T}(\beta) \tilde{\mathbf{q}}(t)$

where $\boldsymbol{\psi}, \tilde{\boldsymbol{\psi}}, \mathbf{q}$ and $\tilde{\mathbf{q}}$ are the mode vector-functions and the corresponding general coordinates of the normal and tangential displacements respectively,

$\mathbf{q}(t)=\left[q_{0}(t), q_{2}(t), q_{3}(t), q_{4}(t), \ldots\right]^{T}$

$\tilde{\mathbf{q}}(t)=\left[\tilde{q}_{2}(t), \tilde{q}_{3}(t), \tilde{q}_{4}(t), \ldots\right]^{T}$

$\boldsymbol{\psi}(\beta)=[1, \cos (2 \beta), \cos (3 \beta), \cos (4 \beta), \ldots]^{T}$

$\tilde{\boldsymbol{\Psi}}(\beta)=[\sin (2 \beta), \sin (3 \beta), \sin (4 \beta), \ldots]^{T}$

The governing equations for the cylindrical shell in free drop can be derived from (2.3) as follows, see Sun et al. ${ }^{[7]}$ for more details,

$\left\{\begin{array}{l}M g-M \ddot{\zeta}=Q_{\zeta} \\ \frac{M}{2} \ddot{\mathbf{q}}+2 D_{3}\left(\mathbf{k}_{2} \ddot{\mathbf{q}}-\mathbf{k}_{1} \ddot{\tilde{\mathbf{q}}}\right)+2 \pi D_{1}\left(\mathbf{q}-\mathbf{k}_{1} \tilde{\mathbf{q}}\right)+2 \pi D_{2}\left(\mathbf{k}_{4} \mathbf{q}-\mathbf{k}_{3} \tilde{\mathbf{q}}\right)=\mathbf{Q}_{q} \\ \frac{M}{2} \ddot{\tilde{\mathbf{q}}}+2 D_{3}\left(\ddot{\tilde{\mathbf{q}}}-\mathbf{k}_{1} \ddot{\mathbf{q}}\right)+2 \pi D_{1}\left(\mathbf{k}_{2} \tilde{\mathbf{q}}-\mathbf{k}_{1} \mathbf{q}\right)+2 \pi D_{2}\left(\mathbf{k}_{2} \tilde{\mathbf{q}}-\mathbf{k}_{3} \mathbf{q}\right)=\mathbf{0}\end{array}\right.$

The first order modes, i.e. $\cos (\beta)$ and $\sin (\beta)$, correspond to the rigid motions of the cylinder, which is considered separately in the first equation of (2.21).

The diagonal matrices $\mathbf{k}_{m}, \mathrm{~m}=1,2,3,4$, and the coefficients $D_{1}, D_{2}$ and $D_{3}$ in (2.21) are defined by

$\mathbf{k}_{m}=\operatorname{diag}\left(k^{m}\right) \quad k=0,2,3,4, \ldots$

$D_{1}=\frac{E h}{2\left(1-v^{2}\right) R}$

$D_{2}=\frac{E h^{3}}{24\left(1-v^{2}\right) R^{3}}$

$D_{3}=\frac{\rho_{s} \pi h^{3}}{24 R}$ 
The non-conservative forces in (2.21) are calculated by (2.10) and (2.11) correspondingly, see Section 2.2. Then the equations (2.9) for a wedge and equations (2.21) for a cylindrical shell are integrated in time by the Newmark method ${ }^{[32]}$. The coupling scheme between fluid and structure solvers is explained in Section 2.3.

\subsection{Hydrodynamic pressure computation by MLM}

As shown in (2.9) and (2.21), the gravity is included in the structure dynamic equations. In terms of hydrodynamic loads, the gravity effect is normally not included in the analytical models (Wagner, MLM or other similar models) ${ }^{[23]}$ for slamming of blunt body with relatively large impact velocity. According to the analysis by Zekri ${ }^{[33]}$, the gravity contribution to the hydrodynamic loads can be estimated using the parameter $\delta=g \sqrt{T /\left(V R_{c}\right)}$, in which $T$ is the duration of the impact, $R_{c}$ is the radius of curvature of the body surface. For slamming of blunt shapes with relatively large impact speed, as investigated in this paper, the impact duration is normally within the order of $\mathrm{O}$ (1e-2)s. This indicates that the parameter is small enough and the gravity effects can be neglected in the leading order for the initial impact phase.

The gravity can be important for shapes which are not blunt with not small deadrise angles. Then the duration of the impact phase is longer and the parameter can be big enough to justify account for gravity. MLM model was developed to deal with such non-blunt shapes, for which the Wagner model is not applicable. The gravity can also be important during the vibration (penetration) phase, when the local velocities of the structure elements become small. Including gravity into impact models is not trivial, even for Wagner-based models, because the boundary condition on the free surface becomes much more complicated. A promising study of this problem was published by Khabakhpasheva et al. ${ }^{[34]}$. For a rigid wedge with 45 degrees deadrise angle entering water with deceleration. Even for relatively large initial speed of entry, $4 \mathrm{~m} / \mathrm{s}$, and deceleration $3 \mathrm{~g}$ the gravity contribution was significant for the phase of impact when the wedge dropped to zero. The MLM with gravity developed in Khabakhpasheva et al. ${ }^{[34]}$ provided the same hydrodynamic force as OpenFoam with gravity. However, this MLM with gravity model has not been applied to hydroelastic impacts yet.

In MLM model, the hydrodynamic pressure is computed by the non-linear Bernoulli equation:

$$
p(y, z, t)=-\rho\left(\dot{\varphi}+\frac{1}{2}|\nabla \varphi|^{2}\right)
$$

where $\varphi(y, z, t)$ is the velocity potential of the flow, and $\rho$ is the water density. Following ${ }^{[23]}$, the velocity potential and the pressure on the wetted body surface are introduced,

$$
\begin{aligned}
& P(y, t)=p(y, f(y, t), t) \\
& \phi(y, t)=\varphi(y, f(y, t), t)
\end{aligned}
$$

By using equations (2.24-26) and the body boundary condition,

$$
-\frac{\partial \varphi}{\partial y} \frac{\partial f}{\partial y}+\frac{\partial \varphi}{\partial z}=\dot{f}
$$


we find

$$
P(y, t)=-\rho\left[\dot{\phi}-\frac{\dot{f} f^{\prime}}{1+f^{\prime 2}} \phi^{\prime}+\frac{1}{2} \frac{\phi^{\prime 2}-\dot{f}^{2}}{1+f^{\prime 2}}\right]
$$

The variable with prime represents the differentiation with respect to $y$. The velocity potential on the wetted body surface $\phi(y, t)$ is approximated by using the Taylor expansion of the classic Wagner potential $\hat{\varphi}(y, 0, t)$ as follows:

$\phi(y, t)=\hat{\varphi}(y, 0, t)+f(y, t) \cdot \widehat{\varphi}_{z}^{\prime}(y, 0, t)$

where $\hat{\varphi}(y, 0, t)$ satisfies the following equations,

$$
\begin{cases}\Delta \hat{\varphi}=0 & (z<0) \\ \widehat{\varphi}=0 & (z=0,|y| \geq c) \\ \frac{\partial \hat{\varphi}}{\partial z}=\dot{f}(y, t) & (z=0,|y|<c) \\ \widehat{\varphi} \rightarrow 0 & \left(y^{2}+z^{2} \rightarrow \infty\right)\end{cases}
$$

Substituting (2.29) into (2.28), the pressure on the wetted body surface is obtained as

$$
P(y, t)=-\rho\left[\dot{\hat{\varphi}}+\hat{\varphi}^{\prime} \frac{\dot{f}^{\prime} f}{1+f^{\prime 2}}+\frac{\hat{\varphi}^{\prime 2}}{2\left(1+f^{\prime 2}\right)}+\frac{\dot{f}^{\prime 2} f^{2}}{2\left(1+f^{\prime 2}\right)}+\ddot{f} f+\frac{1}{2} \dot{f}^{2}\right]
$$

The function $c(t)$, which describes the size of the wetted part of the body surface for a symmetric body, see Fig.1, satisfies the following equation ${ }^{[23]}$,

$$
\int_{0}^{\frac{\pi}{2}} f(c \sin \alpha, t) d \alpha=0
$$

By substituting (2.1)-(2.2) in (2.31) and differentiating the result in time, we find the relations between $\zeta(t)$ and $c(t)$ as

$$
\frac{\pi}{2} \zeta=\int_{0}^{\frac{\pi}{2}}\left[f_{d}(c \sin \alpha, t)\right] d \alpha
$$

and between $\dot{\zeta}(t)$ and $\dot{c}(t)$ as

$$
\frac{\pi}{2} \dot{\zeta}=\int_{0}^{\frac{\pi}{2}}\left[\dot{c} \sin \alpha f_{d}^{\prime}(c \sin \alpha, t)+\dot{f}_{d}(c \sin \alpha, t)\right] d \alpha
$$

Note that $f_{d}(y, t)$ depends on elastic deflection of the body, which is unknown in advance and should be determined together with the hydrodynamic loads.

The mixed boundary-value problem (2.30) for $\hat{\varphi}(y, 0, t)$ is solved in the same way as in Tassin et al. ${ }^{[28]}$, where the total hydrodynamic pressure is divided into two parts that are dependent on velocity and acceleration respectively, i.e. $P(y, t)=P_{v}(y, t)+P_{a}(y, t)$. The results by Tassin et al. 
${ }^{[28]}$ provide, (see equations (22)-(23) in Tassin et al. ${ }^{[28]}$ ),

$$
\begin{aligned}
\frac{P_{v}(y, t)}{-\rho} & =\frac{1}{\pi \sqrt{c(t)^{2}-y^{2}}}\left[-\dot{c}(t) c(t) I_{1}(c(t), t)+I_{2}(y, t) \frac{\dot{f}_{d}^{\prime}\left(f_{d}-\zeta\right)}{1+f_{d}^{\prime 2}}\right] \\
& +\frac{1}{c(t)^{2}-y^{2}} \frac{I_{2}^{2}(y, t)}{2 \pi^{2}\left[1+f_{d}^{\prime 2}\right]}+\frac{\left(\dot{f}_{d}-\dot{\zeta}\right)^{2}}{2}+\frac{\dot{f}_{d}^{\prime 2}\left(f_{d}-\zeta\right)^{2}}{2\left[1+f_{d}^{\prime 2}\right]} \\
\frac{P_{a}(y, t)}{-\rho} & =\ddot{f}_{d} f_{d}+\ddot{\zeta} \zeta-f_{d} \ddot{\zeta}-\ddot{f}_{d} \zeta+\frac{I_{3}(y, t)}{\pi} \sqrt{c(t)^{2}-y^{2}}
\end{aligned}
$$

where the integrals $I_{1}(y, t), I_{2}(y, t)$ and $I_{3}(y, t)$ are given by

$$
\begin{aligned}
& I_{1}(y, t)=P V \int_{0}^{c} \frac{2 \tau \theta(\tau, t) d \tau}{\left(\tau^{2}-y^{2}\right) \sqrt{c^{2}(t)-\tau^{2}}} \\
& I_{2}(y, t)=2 y \cdot P V \int_{0}^{c} \frac{\dot{f}(\tau, t) \sqrt{c(t)^{2}-\tau^{2}}}{\tau^{2}-y^{2}} d \tau \\
& I_{3}(y, t)=P V \int_{0}^{c} \frac{-2 \tau \dot{\theta}(\tau, t)}{\left(\tau^{2}-y^{2}\right) \sqrt{c(t)^{2}-\tau^{2}}} d \tau
\end{aligned}
$$

where

$$
\theta(y, t)=-\int_{0}^{y}\left[\dot{f}_{d}(\tau, t)-\dot{\zeta}\right] d \tau
$$

The forces corresponding to the velocity, $F_{v}$, and the acceleration, $F_{a}$, parts of the pressure are calculated separately. It should be mentioned that the velocity dependent pressure, $P_{v}(y, t)$, given by (2.35) is not integrable at $y=c$ due to the term $1 /\left(c(t)^{2}-y^{2}\right)$. As suggested by Korobkin ${ }^{[23]}$, the integration for the force component $F_{v}$ is only conducted where $|y| \leq c^{*}(t)$, with $c^{*}(t)$ being the solution of $P_{v}(y, t)=0$. The force $Q_{\zeta}$ given by (2.10) is decomposed, following the pressure decomposition, as $Q_{\zeta}=F_{R v}+F_{R a}$, where 


$$
\begin{aligned}
-\frac{F_{R v}(t)}{2 \rho}= & -\int_{0}^{c^{*}} \frac{P_{v}(\tau, t)}{\rho} d \tau \\
& =-\frac{\dot{c} c I_{1}(c)}{\pi} \arcsin \frac{c^{*}(t)}{c(t)}+\int_{0}^{c^{*}} \frac{I_{2}(\tau, t)}{\pi \sqrt{c(t)^{2}-\tau^{2}}} \frac{\dot{f}_{d}^{\prime}(\tau, t) f_{d}(\tau, t)}{1+f_{d}^{\prime 2}(\tau)} d \tau \\
& -\zeta \int_{0}^{c^{*}} \frac{I_{2}(\tau, t)}{\pi \sqrt{c(t)^{2}-\tau^{2}}} \frac{\dot{f}_{d}^{\prime}(\tau, t)}{1+f_{d}^{\prime 2}(\tau, t)} d \tau+\int_{0}^{c^{*}} \frac{1}{c(t)^{2}-\tau^{2}} \frac{I_{2}^{2}(\tau, t)}{2 \pi^{2}\left[1+f_{d}^{\prime 2}(\tau, t)\right]} d \tau \\
& +\int_{0}^{c^{*}} \frac{\dot{f}_{d}^{2}(\tau, t)}{2} d \tau-\dot{\zeta} \int_{0}^{c^{*}} \dot{f}_{d}(\tau, t) d \tau+\frac{\dot{\zeta}^{2}}{2} c^{*}(t)+\int_{0}^{c^{*}} \frac{\dot{f}_{d}^{\prime 2}(\tau, t) f_{d}^{2}(\tau, t)}{2\left[1+f_{d}^{\prime 2}(\tau, t)\right]} d \tau \\
& -\zeta \int_{0}^{c^{*}} \frac{\dot{f}_{d}^{\prime 2}(\tau, t) f_{d}(\tau, t)}{1+f_{d}^{\prime 2}(\tau, t)} d \tau+\zeta^{2} \int_{0}^{c^{*}} \frac{\dot{f}_{d}^{\prime 2}(\tau, t)}{2\left[1+f_{d}^{\prime 2}(\tau, t)\right]} d \tau \\
-\frac{F_{R a}(t)}{2 \rho} & =-\int_{0}^{c} \frac{P_{a}(\tau, t)}{\rho} d \tau \\
& =\int_{0}^{c} \ddot{f}_{d}(\tau, t) f_{d}(\tau, t) d \tau-\ddot{\zeta} \int_{0}^{c} f_{d}(\tau, t) d \tau-\zeta \int_{0}^{c} \ddot{f_{d}}(\tau, t) d \tau+c \ddot{\zeta} \zeta \\
& +\int_{0}^{c} \frac{I_{3}(\tau, t) \sqrt{c(t)^{2}-\tau^{2}}}{\pi} d \tau
\end{aligned}
$$

The force $Q_{q_{k}}$, which corresponds to $k^{\text {th }}$ elastic mode and is given by (2.11), is decomposed as

$$
\begin{aligned}
& Q_{q_{k}}=F_{E v k}+F_{E a k}, \text { where } \\
& -\frac{F_{E v k}}{2 \rho}=-\int_{0}^{c^{*}} \frac{P_{v}(\tau, t) \psi_{k}(\tau) n_{z}}{\rho} d \tau \\
& =-\frac{\dot{c} c I_{1}(c(t), t)}{\pi} \int_{0}^{c^{*}} \frac{\psi_{k}(\tau) n_{z}}{\sqrt{c(t)^{2}-\tau^{2}}} d \tau+\int_{0}^{c^{*}} \frac{I_{2}(\tau, t)}{\pi \sqrt{c(t)^{2}-\tau^{2}}} \frac{\dot{f}_{d}^{\prime}(\tau, t) f_{d}(\tau, t)}{1+f_{d}^{\prime 2}(\tau, t)} \psi_{k} \cdot n_{z} d \tau \\
& -\zeta \int_{0}^{c^{*}} \frac{I_{2}(\tau, t)}{\pi \sqrt{c(t)^{2}-\tau^{2}}} \frac{\dot{f}_{d}^{\prime}(\tau, t)}{1+f_{d}^{\prime 2}(\tau, t)} \psi_{k}(\tau) \cdot n_{z} d \tau+\int_{0}^{c^{*}} \frac{\psi_{k}(\tau) n_{z}}{c(t)^{2}-\tau^{2}} \frac{I_{2}^{2}(\tau, t)}{2 \pi^{2}\left[1+f_{d}^{\prime 2}(\tau, t)\right]} d \tau \\
& +\int_{0}^{c^{*}} \frac{\dot{f}_{d}^{2}(\tau, t) \psi_{k}(\tau) \cdot n_{z}}{2} d \tau-\dot{\zeta} \int_{0}^{c^{*}} \dot{f}_{d}(\tau, t) \psi_{k}(\tau) \cdot n_{z} d \tau+\frac{\dot{\zeta}^{2}}{2} \int_{0}^{c^{*}} \psi_{k}(\tau) \cdot n_{z} d \tau \\
& +\int_{0}^{c^{*}} \frac{\dot{f}_{d}^{\prime 2}(\tau, t) f_{d}^{2}(\tau, t) \psi_{k}(\tau) n_{z}}{2\left[1+f_{d}^{\prime 2}(\tau, t)\right]} d \tau-\zeta \int_{0}^{c^{*}} \frac{\dot{f}_{d}^{\prime 2}(\tau, t) f_{d}(\tau, t) \psi_{k}(\tau) \cdot n_{z}}{1+f_{d}^{\prime 2}(\tau, t)} d \tau \\
& +\zeta^{2} \int_{0}^{c^{*}} \frac{\dot{f}_{d}^{\prime 2}(\tau, t) \psi_{k}(\tau) \cdot n_{z}}{2\left[1+f_{d}^{\prime 2}(\tau, t)\right]} d \tau
\end{aligned}
$$




$$
\begin{aligned}
-\frac{F_{E a k}}{2 \rho} & =-\int_{0}^{c} \frac{P_{a}(\tau, t) \psi_{k}(\tau) n_{z}}{\rho} d \tau \\
& =\int_{0}^{c} \ddot{f}_{d}(\tau, t) f_{d}(\tau, t) \psi_{k}(\tau) n_{z} d \tau-\ddot{\zeta} \int_{0}^{c} f_{d}(\tau, t) \psi_{k}(\tau) n_{z} d \tau \\
& -\zeta \int_{0}^{c} \ddot{f}_{d}(\tau, t) \psi_{k}(\tau) n_{z} d \tau+\ddot{\zeta} \zeta \int_{0}^{c} \psi_{k}(\tau) n_{z} d \tau \\
& +\int_{0}^{c} \frac{I_{3}(\tau, t) \sqrt{c(t)^{2}-\tau^{2}} \psi_{k}(\tau) n_{z}}{\pi} d \tau
\end{aligned}
$$

In order to deal with an arbitrary symmetric body shape, the position, velocity and acceleration of the deformed body surface was discretized by a series of points and interpolated linearly between each point as given in (2.45)-(2.47). The integration is then conducted segment by segment. The number of segments for half side of the body shape used in the simulations in Section 3 is 50 .

$$
\begin{array}{ll}
f_{d}(y, t)=f_{d}\left(y_{n}, t\right)+K_{d n} \cdot\left(y-y_{n}\right), & y_{n} \leq y<y_{n+1} \\
\dot{f}_{d}(y, t)=\dot{f}_{d}\left(y_{n}, t\right)+\Gamma_{d n} \cdot\left(y-y_{n}\right), & y_{n} \leq y<y_{n+1} \\
\ddot{f}_{d}(y, t)=\ddot{f}_{d}\left(y_{n}, t\right)+\Pi_{d n} \cdot\left(y-y_{n}\right), & y_{n} \leq y<y_{n+1}
\end{array}
$$

where

$$
\begin{aligned}
K_{d n} & =\frac{f_{d}\left(y_{n+1}, t\right)-f_{d}\left(y_{n}, t\right)}{y_{n+1}-y_{n}} \\
\Gamma_{d n} & =\frac{\dot{f}_{d}\left(y_{n+1}, t\right)-\dot{f}_{d}\left(y_{n}, t\right)}{y_{n+1}-y_{n}} \\
\Pi_{d n} & =\frac{\ddot{f}_{d}\left(y_{n+1}, t\right)-\ddot{f}_{d}\left(y_{n}, t\right)}{y_{n+1}-y_{n}}
\end{aligned}
$$

The body deformation $f_{d}\left(y_{n}, t\right), f_{d}\left(y_{n+1}, t\right)$ at $y_{n}$ and $y_{n+1}$, and their first and second order time derivatives are calculated by using (2.4) for wedge and (2.16) for cylinder. The values of $c(t)$ and $c^{*}(t)$ are obtained by solving (2.33)-(2.34) with the help of the predicted body position, velocity and acceleration (which is conducted in an iterative manner and will be explained in Section 2.3). More specifically, $c(t)$ is first obtained by solving (2.33) with bisection method, and the initial searching scope is set to be within 10 segments before and after the value in last time step. The threshold of convergence for the bisection searching is chosen to be 1e-5. After obtaining $c(t)$, $c^{*}(t)$ can then be solved directly through (2.34).

For each integral in (2.41)-(2.44), the integration within each segment is calculated by the 5 points Gauss Quadrature. These integrations require the value of $I_{1}(y, t), I_{2}(y, t)$ and $I_{3}(y, t)$ in- 
between the discretized points. They are obtained by linearly interpolating from the adjacent discretized points, which are calculated beforehand. In order to calculate the principal value integrals (i.e. Cauchy principal value $\left.{ }^{[35]}\right)$ such as $I_{1}(y, t), I_{2}(y, t)$ and $I_{3}(y, t)$, following the approach by Tassin et al ${ }^{[28]}$, the transformation in (2.51) is first applied:

$$
P V \int_{0}^{c} \frac{g(\tau, t)}{\left(\tau^{2}-y^{2}\right) \sqrt{c^{2}(t)-\tau^{2}}} d \tau=\int_{0}^{c} \frac{g(\tau, t)-g(y, t)}{\left(\tau^{2}-y^{2}\right) \sqrt{c^{2}(t)-\tau^{2}}} d \tau
$$

where $0<\mathrm{y}<\mathrm{c}$ and $g(y, t)$ represents a function that satisfies Hölder's condition with respect to $y$, which would make sure that the improper integrals in the right-hand side of (2.51) converges.

By using (2.51) and the body surface discretization in (2.45)-(2.47), at a particular discretized point such as $y_{k}$, the integrals $I_{1}\left(y_{k}, t\right), I_{2}\left(y_{k}, t\right)$ and $I_{3}\left(y_{k}, t\right)$ are computed as follows:

$$
\begin{aligned}
& I_{1}\left(y_{k}, t\right)=\sum_{n=1}^{M-1} \int_{y_{n}}^{y_{n+1}} \frac{2 \tau \theta(\tau, t)-2 y_{k} \theta\left(y_{k}, t\right)}{\left(\tau^{2}-y_{k}^{2}\right) \sqrt{c^{2}(t)-\tau^{2}}} d \tau+\int_{y_{M}}^{c} \frac{2 \tau \theta(\tau, t)-2 y_{k} \theta\left(y_{k}, t\right)}{\left(\tau^{2}-y_{k}^{2}\right) \sqrt{c^{2}(t)-\tau^{2}}} d \tau \\
& I_{2}\left(y_{k}, t\right)=\pi y_{k} \dot{\zeta} \\
& +2 y_{k} c(t)^{2}\left[\sum_{n=1}^{M-1} \int_{y_{n}}^{y_{n+1}} \frac{\dot{f}_{d}(\tau)-\dot{f}_{d}\left(y_{k}\right)}{\left(\tau^{2}-y_{k}^{2}\right) \sqrt{c(t)^{2}-\tau^{2}}} d \tau+\int_{y_{M}}^{c} \frac{\dot{f}_{d}(\tau)-\dot{f}_{d}\left(y_{k}\right)}{\left(\tau^{2}-y_{k}^{2}\right) \sqrt{c(t)^{2}-\tau^{2}}} d \tau\right] \\
& I_{3}\left(y_{k}, t\right)=\sum_{n=1}^{M-1} \int_{y_{n}}^{y_{n+1}} \frac{2 y_{k} \dot{\theta}\left(y_{k}, t\right)-2 \tau \dot{\theta}(\tau, t)}{\left(\tau^{2}-y_{k}^{2}\right) \sqrt{c(t)^{2}-\tau^{2}}} d \tau+\int_{y_{M}}^{c} \frac{2 y_{k} \dot{\theta}\left(y_{k}, t\right)-2 \tau \dot{\theta}(\tau, t)}{\left(\tau^{2}-y_{k}^{2}\right) \sqrt{c(t)^{2}-\tau^{2}}} d \tau
\end{aligned}
$$

Similarly, the integral $\theta(y, t)$ required in the computation of (2.52)-(2.54) is first calculated at each discretized point beforehand:

$$
\begin{aligned}
& \theta\left(y_{k}, t\right)=\dot{\zeta} y_{k}-\sum_{n=1}^{k-1}\left[\dot{f}_{d}\left(y_{n}, t\right) \cdot\left(y_{n+1}-y_{n}\right)+\frac{1}{2} \Gamma_{d n} \cdot\left(y_{n+1}-y_{n}\right)^{2}\right] \\
& \dot{\theta}\left(y_{k}, t\right)=\ddot{\zeta} y_{k}-\sum_{n=1}^{k-1}\left[\ddot{f}_{d}\left(y_{n}, t\right) \cdot\left(y_{n+1}-y_{n}\right)+\frac{1}{2} \Pi_{d n} \cdot\left(y_{n+1}-y_{n}\right)^{2}\right]
\end{aligned}
$$

and the value between the discretized points (i.e. $y \in\left[y_{n}, y_{n+1}\right]$ ) is expressed as the linear interpolation among adjacent points:

$$
\begin{aligned}
& \theta(y, t)=\theta\left(y_{k}, t\right)+\dot{\zeta} \cdot\left(y-y_{k}\right)-\left[\dot{f}_{d}\left(y_{k}, t\right) \cdot\left(y-y_{k}\right)+\frac{1}{2} \Gamma_{d k} \cdot\left(y-y_{k}\right)^{2}\right] \\
& \dot{\theta}(y, t)=\dot{\theta}\left(y_{k}, t\right)+\ddot{\zeta} \cdot\left(y-y_{n}\right)-\left[\ddot{f}_{d}\left(y_{k}, t\right) \cdot\left(y-y_{k}\right)+\frac{1}{2} \Pi_{d k} \cdot\left(y-y_{k}\right)^{2}\right]
\end{aligned}
$$

The integrations of (2.52)-(2.54) take different forms depending whether $y_{k}$ is within the scope of the current integration segment. Specifically, the integrations from $y_{n}$ to $y_{n+l}$, which are denoted 
as $I_{1 n}, I_{2 A n}, I_{2 B n}$ and $I_{3 n}$ (note that $I_{2 n}=I_{2 A n}+I_{2 B n}$ ), are calculated as in (2.59)-(2.61), and the integration from $y_{M}$ to $c I_{1 c}, I_{2 A c}, I_{2 B c}$ (note that $I_{2 c}=I_{2 A c}+I_{2 B c}$ ) and $I_{3 c}$ are conducted by the same procedure.

$$
\begin{gathered}
I_{1 n}\left(y_{k}, t\right)=\int_{y_{n}}^{y_{n+1}}\left[\frac{-K_{d n} \tau}{\sqrt{c(t)^{2}-\tau^{2}}}+\frac{2\left(\dot{\zeta}-\dot{f}_{d}\left(y_{n}, t\right)+K_{d n} y_{n}\right)}{\sqrt{c(t)^{2}-\tau^{2}}}\right] \\
\left.+\frac{2 \theta\left(y_{n}, t\right)-2 \dot{\zeta} y_{n}+2 \dot{f}_{d}\left(y_{n}, t\right) y_{n}-K_{d n} y_{n}^{2}-K_{d n} y_{k}^{2}}{\left(\tau+y_{k}\right) \sqrt{c(t)^{2}-\tau^{2}}}\right] d \tau \\
I_{2 A n}\left(y_{k}, t\right)=\int_{y_{n}}^{y_{n+1}} \frac{K_{n}}{\left(\tau+y_{k}\right) \sqrt{c(t)^{2}-\tau^{2}}} d \tau \\
I_{2 B n}\left(y_{k}, t\right)=\int_{y_{n}}^{y_{n+1}} \frac{\left(\dot{f}_{d n}-K_{n} y_{n}\right)}{\sqrt{c(t)^{2}-\tau^{2}} d \tau+\int_{y_{n}}^{y_{n+1}} \frac{K_{n} \tau}{\sqrt{c(t)^{2}-\tau^{2}}} d \tau} d K_{n} y_{k}^{2} \\
+\int_{y_{n}}^{y_{n+1}} \frac{\left(\tau+y_{k}\right) \sqrt{c(t)^{2}-\tau^{2}}}{\left(\tau+y_{k}\right) \sqrt{c(t)^{2}-\tau^{2}}} \\
I_{3 n}\left(y_{k}, t\right)=\int_{y_{n}}^{y_{n+1}}\left[\frac{\Gamma_{d n} \tau}{\sqrt{c(t)^{2}-\tau^{2}}}+\frac{2\left[-\ddot{\zeta}+\ddot{f}_{d}\left(y_{n}, t\right)-\Gamma_{d n} y_{n}\right]}{\sqrt{c(t)^{2}-\tau^{2}}}\right. \\
+\frac{-2 \theta\left(y_{n}, t\right)+2 \ddot{\zeta} y_{n}-2 \ddot{f_{d}}\left(y_{n}, t\right) y_{n}+\Gamma_{n n} y_{n}^{2}+\Gamma_{d n} y_{k}^{2}}{(\tau)}
\end{gathered}
$$

If $y_{k} \notin\left[y_{n}, y_{n+1}\right]$, the integration is conducted as follows:

$$
\begin{aligned}
I_{1 n}\left(y_{k}, t\right) & =\int_{y_{n}}^{y_{n+1}}\left[\frac{\left(2 \theta\left(y_{n}, t\right)-2 \dot{\zeta} y_{n}+2 \dot{f}_{d}\left(y_{n}, t\right) \cdot y_{n}-K_{d n} y_{n}^{2}\right) \tau}{\left(\tau^{2}-y_{k}^{2}\right) \sqrt{c(t)^{2}-\tau^{2}}}\right. \\
& +\frac{2\left[\dot{\zeta}-\dot{f}_{d}\left(y_{n}, t\right)+K_{d n} y_{n}\right]}{\sqrt{c(t)^{2}-\tau^{2}}}+\frac{2 y_{k}^{2}\left[\dot{\zeta}-\dot{f}_{d}\left(y_{n}, t\right)+K_{d n} y_{n}\right]-2 y_{k} \theta\left(y_{k}, t\right)}{\left(\tau^{2}-y_{k}^{2}\right) \sqrt{c(t)^{2}-\tau^{2}}} \\
& \left.-\frac{K_{d n} \tau^{3}}{\left(\tau^{2}-y_{k}^{2}\right) \sqrt{c(t)^{2}-\tau^{2}}}\right] d \tau \\
I_{2 A n}\left(y_{k}, t\right) & =\int_{y_{n}}^{y_{n+1}} \frac{\dot{f}_{d}\left(y_{n}, t\right)-K_{d n} y_{n}-\dot{f}_{d}\left(y_{k}, t\right)}{\left(\tau^{2}-y_{k}^{2}\right) \sqrt{c(t)^{2}-\tau^{2}}} d \tau+\int_{y_{n}}^{y_{n+1}} \frac{K_{d n} \tau}{\left(\tau^{2}-y_{k}^{2}\right) \sqrt{c(t)^{2}-\tau^{2}}} d \tau
\end{aligned}
$$




$$
\begin{aligned}
& I_{2 B n}\left(y_{k}, t\right)=\int_{y_{n}}^{y_{n+1}} \frac{\left[\dot{f}_{d}\left(y_{n}, t\right)-K_{d n} y_{n}\right] \tau^{2}}{\left(\tau^{2}-y_{k}^{2}\right) \sqrt{c(t)^{2}-\tau^{2}}} d \tau+\int_{y_{n}}^{y_{n+1}} \frac{K_{d n} \tau^{3}}{\left(\tau^{2}-y_{k}^{2}\right) \sqrt{c(t)^{2}-\tau^{2}}} d \tau \\
& -\int_{y_{n}}^{y_{n+1}} \frac{\dot{f}_{d}\left(y_{k}, t\right) y_{k}^{2}}{\left(\tau^{2}-y_{k}^{2}\right) \sqrt{c(t)^{2}-\tau^{2}}} d \tau \\
& I_{3 n}\left(y_{k}, t\right)=\int_{y_{n}}^{y_{n+1}}\left[\frac{\left(-2 \dot{\theta}\left(y_{k}, t\right)+2 \ddot{\zeta} y_{n}-2 \ddot{f}_{d}\left(y_{n}, t\right) y_{n}+\Gamma_{d n} y_{n}^{2}\right) \tau}{\left(\tau^{2}-y_{k}^{2}\right) \sqrt{c(t)^{2}-\tau^{2}}}\right. \\
& +\frac{2\left(-\ddot{\zeta}+\ddot{f}_{d}\left(y_{n}, t\right)-\Gamma_{d n} y_{n}\right)}{\sqrt{c(t)^{2}-\tau^{2}}}+\frac{2 y_{k}{ }^{2}\left(-\ddot{\zeta}+\ddot{f}_{d}\left(y_{n}, t\right)-\Gamma_{d n} y_{n}\right)+2 y_{k} \dot{\theta}\left(y_{k}, t\right)}{\left(\tau^{2}-y_{k}{ }^{2}\right) \sqrt{c(t)^{2}-\tau^{2}}} \\
& \left.+\frac{\Gamma_{d n} \tau^{3}}{\left(\tau^{2}-y_{k}^{2}\right) \sqrt{c(t)^{2}-\tau^{2}}}\right] d \tau
\end{aligned}
$$

The integrals in (2.60)-(2.66) are evaluated analytically.

\subsection{Coupling of fluid and structural solvers}

The hydrodynamic forces expressed by (2.41)-(2.44) require the position, velocity and acceleration of the structure, which are unknown for the next time step. There are two ways of handling this fully coupled Fluid and Structure Interaction problem. The first approach is to combine the component (2.42) of the hydrodynamic force, which depends on the acceleration of the structure, with the structural inertia terms in equations (2.9) and (2.21). The component (2.41) of the hydrodynamic force, which depends on the shape of the structure and its velocity, is considered as the forcing term in the structural equations. The resulting nonlinear governing equations can be solved by Runge-Kutta method or other time-marching methods. This monolithic approach combines the fluid and structure dynamics into one single set of equations, which are integrated in time without an interface between the solvers and iterations at each time step. The coupling matrix is the added-mass matrix in the original Wagner model for elastic bodies. The corresponding matrix in the MLM is more complicated, see (2.42) and (2.44), but also has a meaning of the added-mass matrix with coupling elastic modes and rigid motions. This added-mass matrix should be calculated at each time step, which could be time consuming. But the benefit is that there is no need for iterations (which is needed in a partitioned approach as shown below) at each time step in a monolithic approach. The convergence property for different structures and impact conditions by using the above mentioned monolithic approach would need detailed investigation, which is planned for the study in the near future.

Alternatively, the partitioned approach can offer more flexibility of utilizing suitable solvers for each computational domain, whilst the resultant subsystems for each part are well conditioned. This type of approach has been successfully used for many CFD based FSI studies ${ }^{[8,36,37]}$. One of the most commonly used partitioned FSI strategy is the Dirichlet-Neumann Coupling with Aitken Relaxation, which is proved to be simple and efficient ${ }^{[29]}$.

More specially, the fluid and structure interaction process was implemented in a prediction- 
correction manner. More specifically, from $t_{n-1}$ to $t_{n}$ (assuming that the structure motion has been obtained), the structure position is first predicted based on constant acceleration assumption using the structure motion value at $t_{n-1} ;$ secondly, the hydrodynamic pressure along the wetted surface can be computed by the procedure given in Section 2.2; thirdly, the position of the structure could be updated using the newly calculated pressure via Newmark method; after that the updated structure position would be compared with the previous prediction, if the difference between the predicted and updated structure positions is below the pre-set criteria, i.e. 1e-6 in this study, the computation is deemed to be converged and proceeding to the next time step, otherwise, the structure position is further corrected by the so-called Aitken relaxation method ${ }^{[38]}$ (by utilizing the information from both prediction and the updated structure position to improve the convergence performance) and then the abovementioned iteration would be repeated until the convergence criteria is met. This process can be illustrated by Fig. 3. The details of this Fluid and Structure Interaction process can be found in $\operatorname{ref}^{[8]}$.

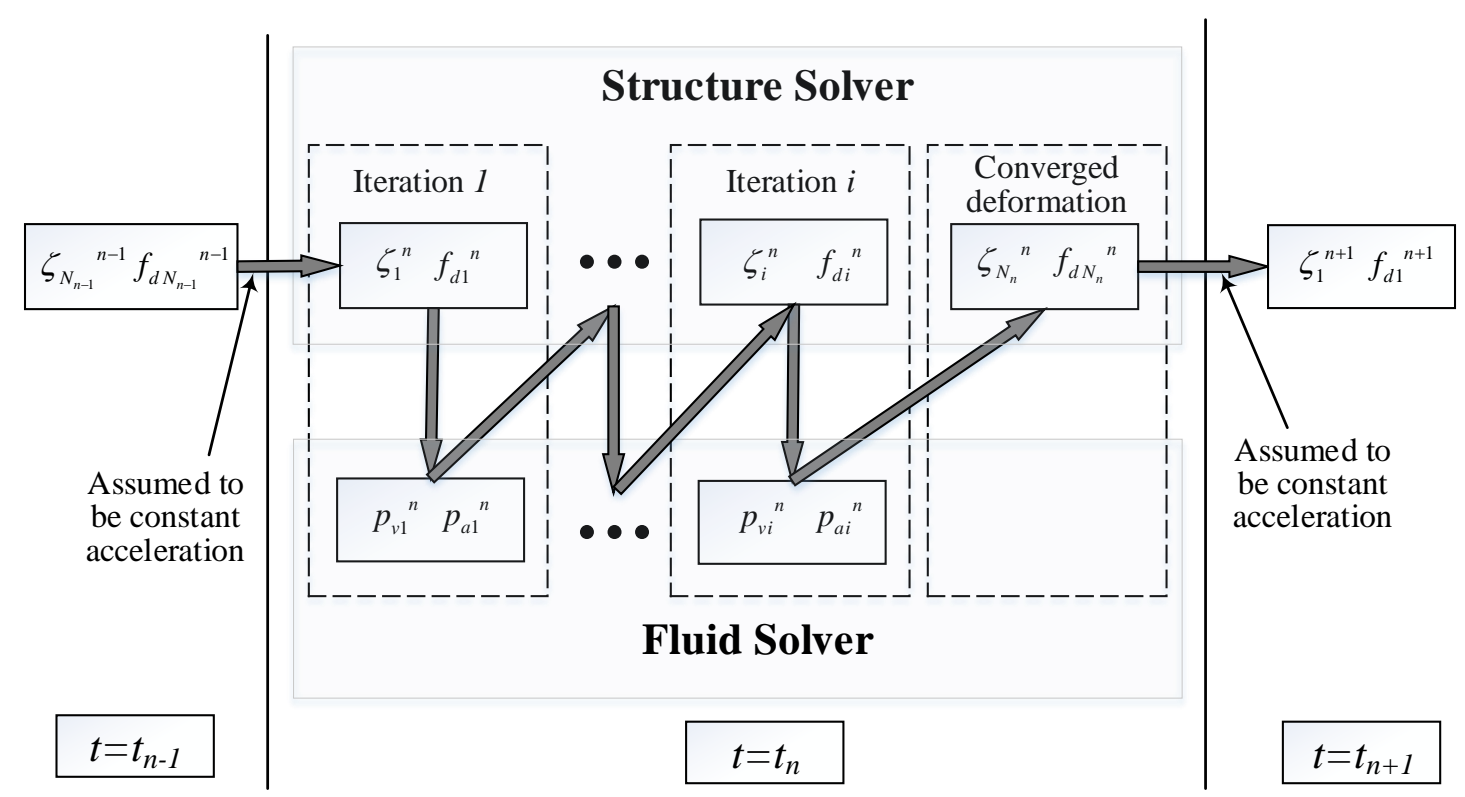

Fig. 3 The Fluid and Structure Interaction process

\section{Results and discussion}

\subsection{Wedge entry with constant velocity}

The performance of the MLM for evaluation of elastic deformation is presented in this section for flexible wedge entry with constant speed. The bottom plating are simply supported on both sides. Parameters of this simulation correspond to case 6 of Table 1. Calculations by the MLM are performed only for the impact stage before the wedge is completely wetted.

The deflection of the beam midpoint is shown in Fig.4. Fig.4(a) shows that the MLM based model provides better match to CFD simulation ${ }^{[39]}$ compared with the Wagner based model, see Korobkin and Khabakhpasheva ${ }^{[40]}$ before the wedge is completely wetted. This demonstrates the better accuracy of MLM based model, which accounts for non-linear terms in the Bernoulli equation 
for hydrodynamic pressure ${ }^{[23]}$.

For the computations after the first peak, which corresponds to the flow after the separation from the wedge edge, the analytical models (both MLM and Wagner) are not applicable with their current forms. Some researchers ${ }^{[40,41]}$ have proposed the virtual boundary technique to extend the applicability of analytical model beyond flow separation, which is used to produce the prediction after $0.14 \mathrm{~s}$ for the Wagener based model in Fig. 4(a). The general trend of the prediction is satisfactory compared with CFD, but the determination of the virtual boundary is heuristic ${ }^{[42]}$ and the theoretical justification of this kind of approach is not yet clear.

The effects of time step and mode number are shown in Fig.4 (b), (c) and (d), respectively. It is clear that within the chosen range of time steps and mode numbers, the deflections by MLM, see. Fig. 4 (b) and (c) show good convergence. Three modes and time step $0.0001 \mathrm{~s}$ were chosen for the following simulations. The strains, which are more sensitive to the mode number than deflections, are also well predicted by using three elastic modes, see Fig. 4(d).

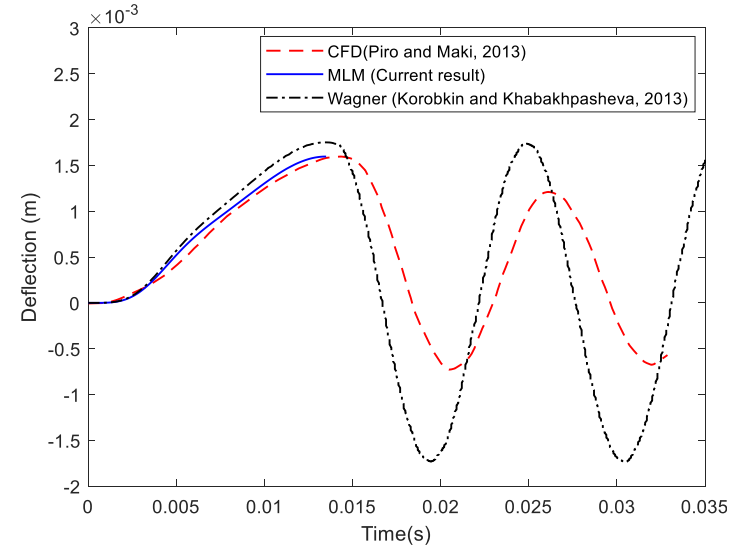

(a)

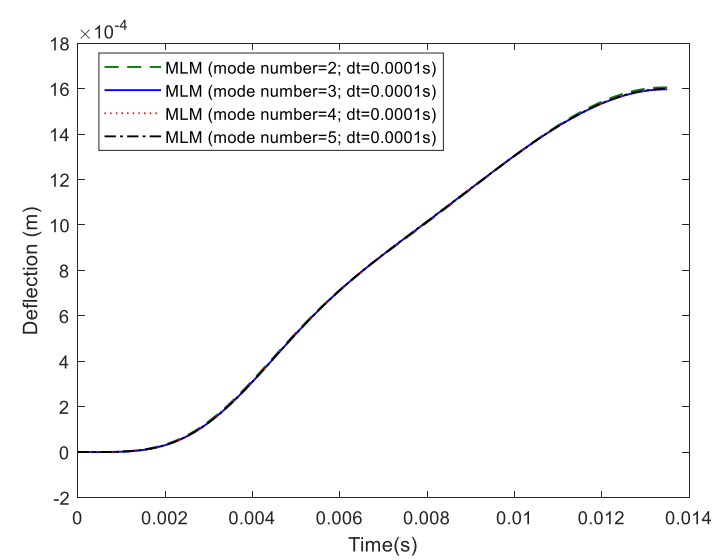

(c)

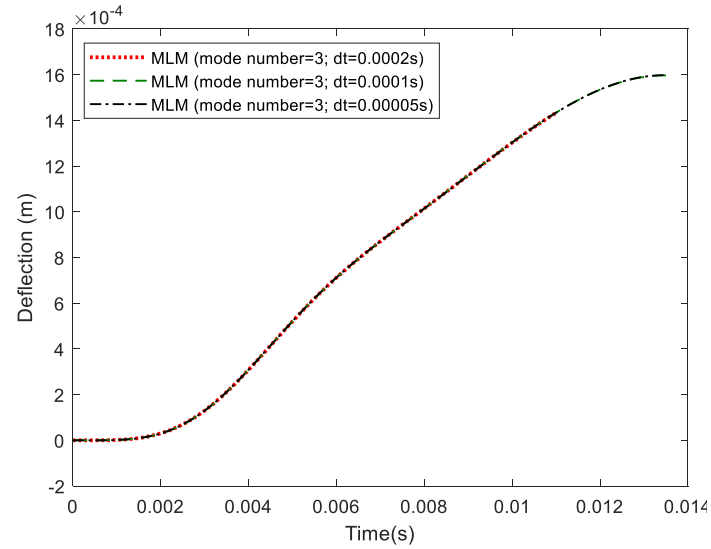

(b)

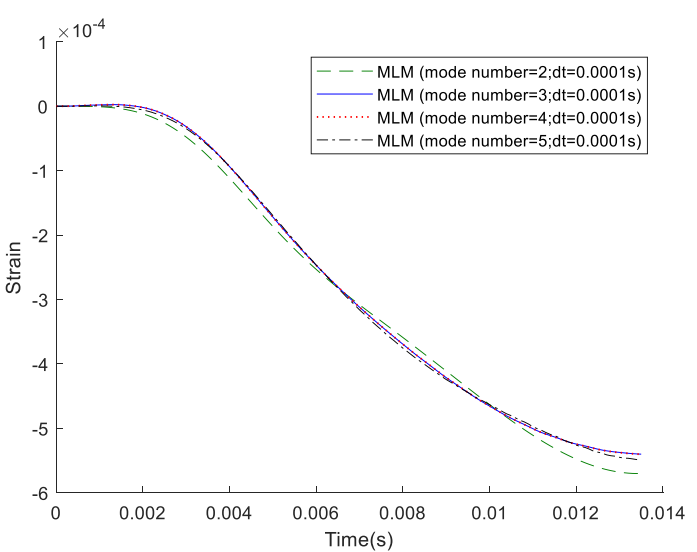

(d)

Fig. 4 The deflections and strains at the beam midpoint for wedge entry with constant velocity (corresponding to Case 6 in Table 1, (a) deflections computed by CFD, MLM and Wagner models;

(b) deflections by MLM computed with different time steps; (c) deflections by MLM with different number of elastic mode; (d) strain at the midpoint by MLM with different number of elastic mode 


\subsection{Free-drop of elastic wedge}

In order to further investigate the performance of the MLM compared with other theoretical models, numerical and experimental results, several cases of free falling elastic wedge are computed. The impact forces by MLM are compared with experimental ${ }^{[43]}$ and available theoretical results ${ }^{[21]}$. The parameters of simulations are given in Table 1. As shown in Fig. 5, the Wagner model without considering the structure deformation (black dotted line) is unable to capture the dynamic features of the hydrodynamic forces during slamming. The hydroelastic computations based on both MLM and Wagner models ${ }^{[21]}$ provide qualitatively acceptable predictions compared with experimental results.

More specifically, the present model accounts for interactions between elastic and rigid motions directly, see equation (2.9) and through the hydrodynamic pressure, whilst the equation of the rigid-body motion in Shams and Porfiri [21] (equation (12) of that paper) does not. This interaction is important in free-fall problems. Comparison of the vertical forces acting on the flexible wedges in free fall, see Fig. 5, where the hydrodynamic forces are both computed by the Wagner model but the structural model accounts (green lines) and does not account (dash-dotted lines) for interaction of elastic and rigid motions, shows that this interaction is important for the prediction of the wedge dynamics, i.e. the vibration frequency of the loads. Furthermore, compared to the results by Wagner model (green lines in Fig. 5), the amplitude of the load vibration predicted by the MLM model (red lines in Fig. 5) tends to be closer to the experimental values shown by blue lines. Therefore, retaining the non-linear term of the Bernoulli equation within MLM, which was done to improve the accuracy of hydrodynamic load prediction, is also beneficial to computation of fluid and structure interaction.

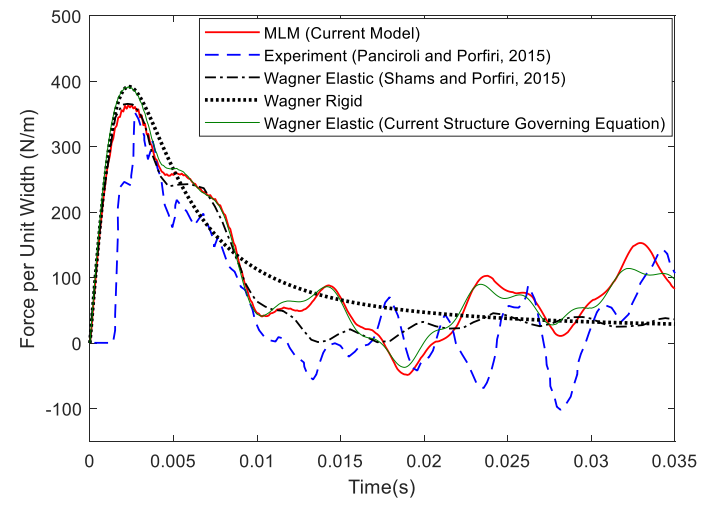

(a) Entry speed $1.87 \mathrm{~m} / \mathrm{s}$

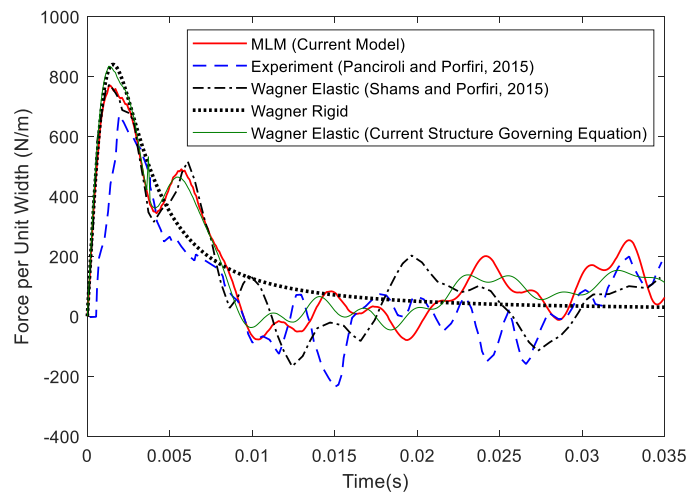

(b) Entry speed $2.77 \mathrm{~m} / \mathrm{s}$ 


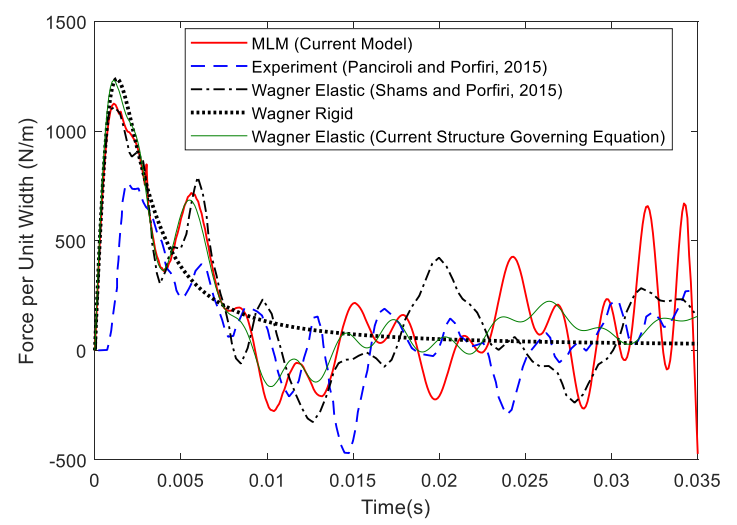

(c) Entry speed $3.38 \mathrm{~m} / \mathrm{s}$

Fig. 5. The vertical forces acting on the elastic wedges in cases 1(a), 2(b) and 3(c) from Table 1 computed and measured in experiments as functions of time.

For the free-drop elastic wedge impact, see Fig. 5(a), both MLM model and the model from ${ }^{[21]}$ predict almost the same value of the force first peak and its rise time for entry speed $1.87 \mathrm{~m} / \mathrm{s}$. The computed rise time of the first peak matches well the experimental one. However, for larger entry speeds, $2.77 \mathrm{~m} / \mathrm{s}$ in Fig. 5(b) and $3.38 \mathrm{~m} / \mathrm{s}$ in Fig. 5(c), both models highly overestimate the peak of the force.

It seems that the measured peak values in experiments do not show a clear rising trend with increasing entry velocities as expected. The experimental peak forces for entry speeds of $2.77 \mathrm{~m} / \mathrm{s}$ and $3.38 \mathrm{~m} / \mathrm{s}$ are about $670 \mathrm{~N} / \mathrm{m}$ and $760 \mathrm{~N} / \mathrm{m}$ respectively, which is not as significant as expected. Actually, as stated in Panciroli and Porfiri ${ }^{[43]}$, the impact speeds in these experiments are about $10 \%$ smaller than theoretical predictions for the corresponding drop heights due to the friction in the guiding rails and air resistance before the impact. This indicates that the friction may also affect the entry velocity during the water entry stage, which could result in a reduction of slamming force exerted on the wedge. This effect could be more significant for larger velocity since friction force is proportional to velocity, which is consistent with the abovementioned measured data in experiments (i.e. for larger entry velocities, the peak impact forces show larger difference from the expected values).

After the first peak, i.e. within the oscillation period (after roughly $0.01 \mathrm{~s}$ ), both. the MLM model and that from Shams and Porfiri ${ }^{[21]}$ ) can "qualitatively capture the oscillations in the hydrodynamic loading", see Shams and Porfiri ${ }^{[21]}$. Generally, the current model produces relatively better results in terms of the local peak/trough values and frequency of the oscillation, but both of the models cannot closely reproduce the local peak values and their corresponding phases. Therefore, a very detailed comparison between the time histories of computed and experimental results (such as infinity norm or two-norm) are not expected to provide a clear picture of the models' performance. Nevertheless, the comparisons of some local quantitative characters between both numerical models and experimental ones are helpful to show the advantages of the current model, in which the nonlinear terms are included in the hydrodynamic computation and the interaction between elastic and rigid-body motions are accounted.

For example, in the case of $1.87 \mathrm{~m} / \mathrm{s}$ entry velocity, the experimental difference between maximum peak and trough within the oscillation period, which is defined as "max amplitude" 
hereafter, is about $244 \mathrm{~N} / \mathrm{m}$. The predictions from the MLM model and the model by Shams and Porfiri ${ }^{[21]}$ are about $200 \mathrm{~N} / \mathrm{m}$ and $48 \mathrm{~N} / \mathrm{m}$, respectively. The MLM model shows a better match with the experimental results. In the case of $2.77 \mathrm{~m} / \mathrm{s}$ entry velocity, both numerical models produce similar max amplitudes, $331 \mathrm{~N} / \mathrm{m}$ and $369 \mathrm{~N} / \mathrm{m}$ for current and Shams and Porfiri [21] models respectively, which are close to the experimental one $(435 \mathrm{~N} / \mathrm{m})$, but the oscillation frequency by the current model is closer to the experimental one. The case of $3.38 \mathrm{~m} / \mathrm{s}$ entry velocity show a very similar pattern, i.e. although the max amplitudes are similar, the oscillation frequency from the current match better with the experimental result.

In terms of the computational efficiency, for a typical computation with time step $0.0001 \mathrm{~s}$, time interval of $0.035 \mathrm{~s}$, and 3 elastic modes, see Fig. 5 , the computational time (with CPU $3.60 \mathrm{GHz} / \mathrm{RAM}$ $16 \mathrm{~GB}$ ) is about 65 secs with 5-6 FSI iterations for each time step. The present model needs longer simulation time compared with the Wagner-based model of [21], but this model was designed to accurately treat elastic shapes even with both moderate deadrise angles and moderate penetration depth, where the application of Wagner-based models could be questionable. At the same time our model is more computationally efficient than BEM models and more complicated CFD-based models.

To validate the MLM model, the strain at different positions of a flexible wedges are compared with both SPH (Smoothed Particle Hydrodynamics) simulations and another experimental results [44]. As shown in Fig. 6-7, the developed model can successfully predict the time history of the deformation during slamming. Generally speaking, for capturing the dynamic features of the elastic vibration, especially at the later stage of slamming, the SPH model was not as effective as the analytical models, namely the deformation predicted by SPH model tended to be flatter than experimental and analytical results. Moreover, the Wagner based model tends to over predict the strains compared with experimental results, whilst the prediction by the current MLM based model provided a better match. However, similarly to the force results, both analytical and SPH models are unable to exactly reproduce the experimental results. For the strains close to the wedge tip, see Fig. 6(a) and 7(a), the absolute value of the strains by MLM are smaller than those from the experiment and other models.

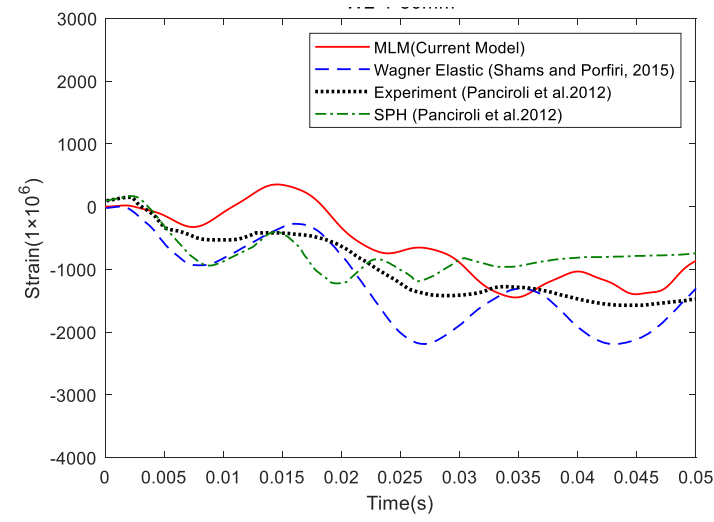

(a)

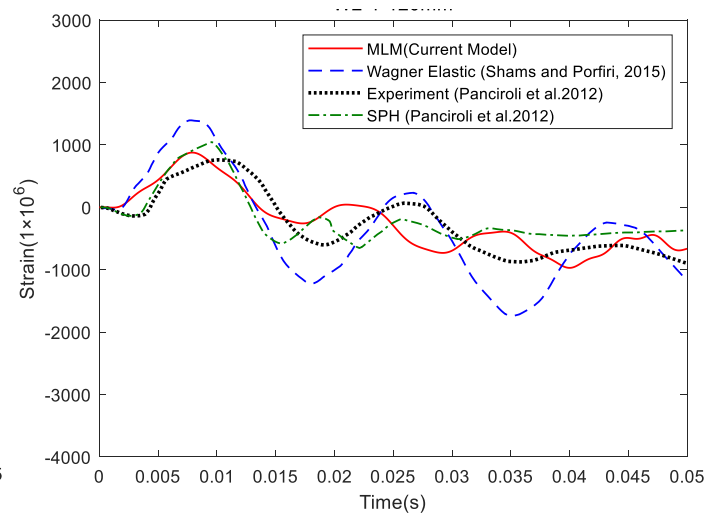

(b)

Fig. 6 The strains in the wedge beam for the case 4 in Table 1 at distances $30 \mathrm{~mm}$ (a) and $120 \mathrm{~mm}$ (b) from the wedge tip. 


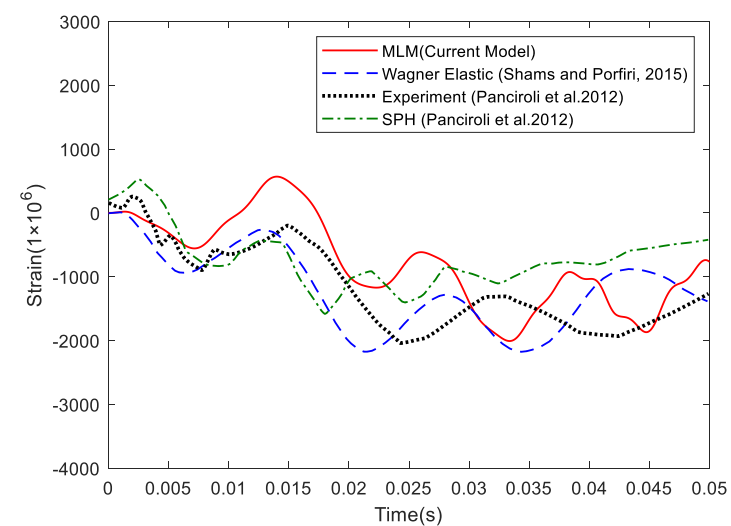

(a)

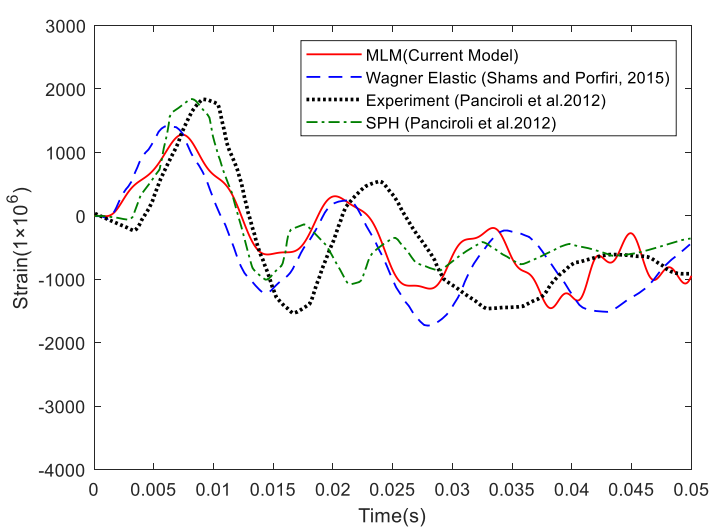

(b)

Fig. 7 The strains in the wedge beam for the case 5 in Table 1 at distances $30 \mathrm{~mm}$ (a) and $120 \mathrm{~mm}$ (b) from the wedge tip.

Importance of hydroelasticity in water impact problems can be quantified using the Dynamic Amplification Factor (DAF), see Faltinsen ${ }^{[45]}$. The DAF shows the increase of dynamic response of a structure with respect to the response predicted by the quasi static interaction model. The DAF depends on the ratio between the duration of the impact and the first wet natural period of the structure, see Fig. 17 in Faltinsen ${ }^{[45]}$. This ratio is proportional to the deadrise angle and inversely proportional to the impact velocity for an elastic wedge. A small ratio means that hydroelasticity is important and a large ratio, greater than 2, means that hydroelasticity is not important. Note that wet natural periods of elastic structure is several times longer than the corresponding dry natural periods. In the five cases 1-5 from Table 1, these ratios are estimated as $0.1235,0.0834,0.0683,0.0874$ and 0.0699 , respectively. Therefore, the cases in the present study are all within the scope where the hydroelasticity effect is significant. The de-coupled or simply rigid-body computations cannot properly capture fluid-structure interaction during these slamming events.

\subsection{Free-drop of cylindrical shell}

In this section, the performance of the developed model for non-wedge structures, i.e. cylindrical shell is investigated. The parameters used in the simulation are provided in Table 2. Fig. 8 shows the comparison of strain time histories at different positions on the shell with experimental results ${ }^{[46]}$, namely the positions with the central angles of $0,10,20$ and 30 degrees respectively starting from the lowest point of the shell. Three elastic modes were used in the simulation.
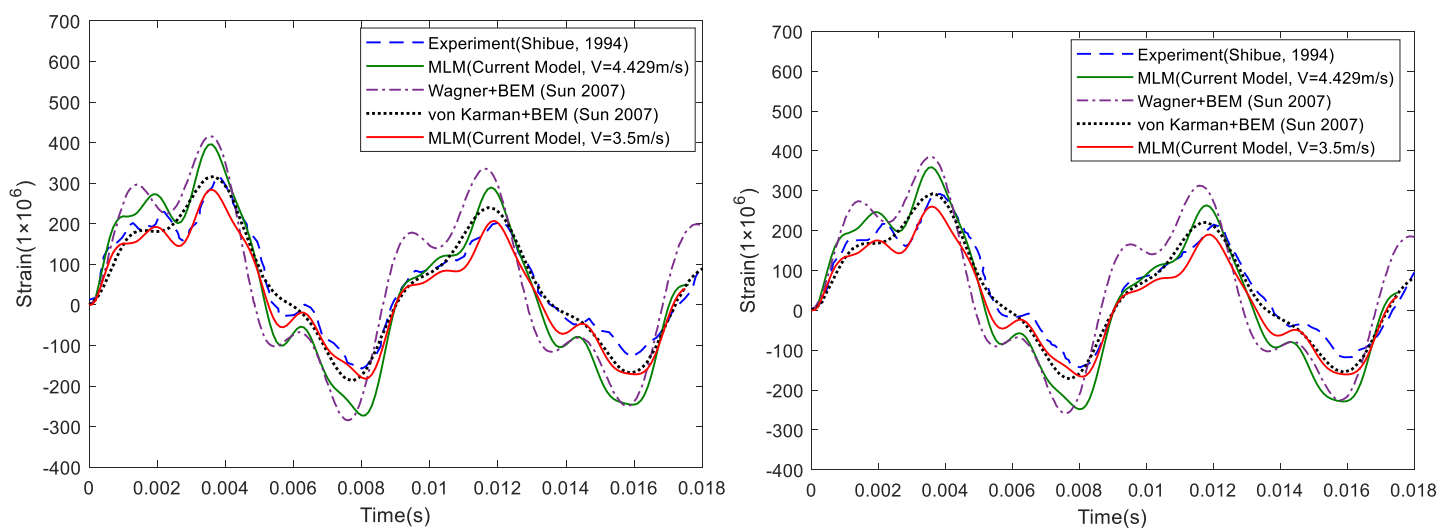
(a)

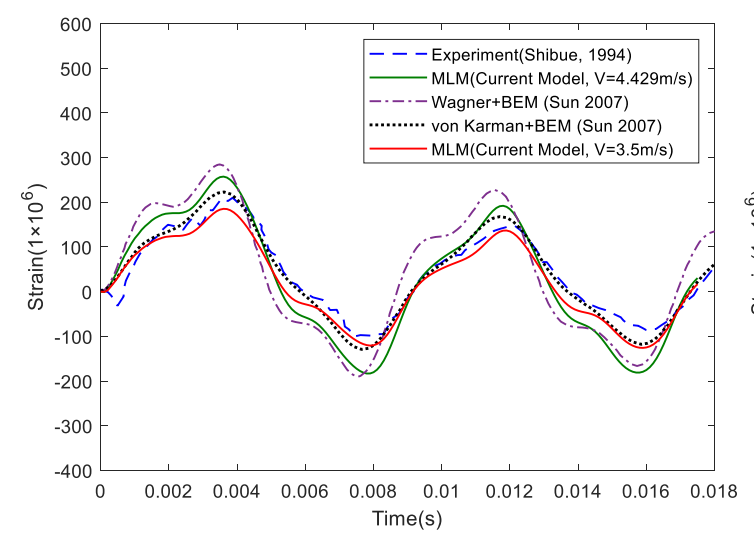

(c) (b)

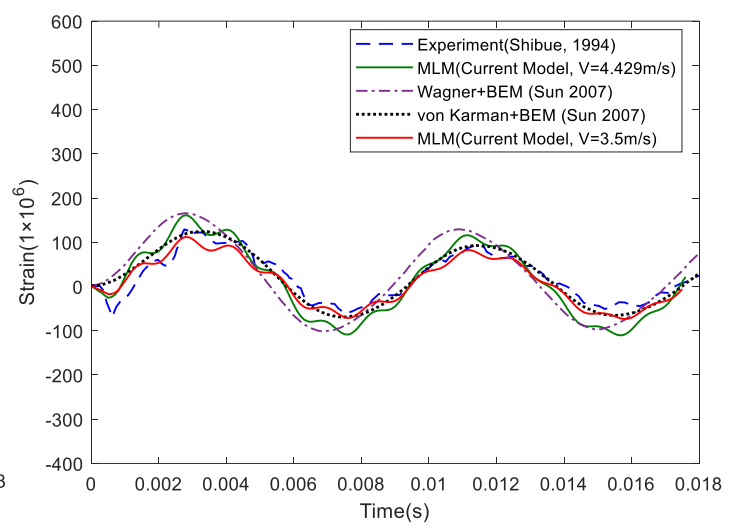

(d)

Fig. 8 The strains in the cylindrical shell at 0 (a), 10 (b), 20 (c) and 30 (d) degree central angles as functions of time.

As shown in Fig. 8, the phase characteristics and vibration details of the dynamic responses can be accurately captured by the developed model, but the magnitude of the strain peak value was higher than that in the experiments. The successful capture of the vibration phase indicates that the structural parameters used in the simulation (e.g. mass, Young's modulus etc.) are consistent with those in the experiments. By further examining the conditions of the experiments ${ }^{[46]}$, we may conclude that the three-dimensional effects were negligible in the experiment because the flow was constrained within the length of the cylinder, i.e. $600 \mathrm{~mm}{ }^{[46]}$ which guaranteed the two dimensional flow under the cylinder. But similar to the wedge dropping experiment in Section 3.2, the shell is also attached to a vertical guiding rail in the experiment ${ }^{[46]}$, which means the entry velocity is very likely to be below the theoretical value (i.e. calculated by $\sqrt{2 g H})$ for a chosen falling height $(\mathrm{H}=1 \mathrm{~m}$ in this case) due to the friction and air resistance effects. But unlike the elastic wedge experiment ${ }^{[43]}$ mentioned above, the exact entry velocity was not measured and reported for this experiment ${ }^{[46]}$. The lack of the measurement data about impact velocity and the pressure distribution along the cylinder bottom makes it difficult to confirm whether the larger calculated peak values were caused by the over prediction of pressure using the developed model or by the inconsistence of the simulation parameters with experiment through ignoring the frictional force of the sliding system.

Dependence of the elastic strains in the shell on initial impact velocity was studied by Ionina and Korobkin ${ }^{[47]}$, see also the paper by Ionina ${ }^{[48]}$ with more details of the analysis. The Wagner model of impact was used with up to 15 elastic modes and the model of the shell, which accounts for extensibility of the neutral surface of the shell. Calculations were performed for the conditions of the experiments in ${ }^{[46]}$ with different values of the initial impact velocity. The formula for the maximum strain $\varepsilon_{\max }$ (in microstrains) at the lowest point of the shell during the impact was derived, $\varepsilon_{\max }=14.386 V^{2}+45.139 \mathrm{~V}$, where $V$ is the initial velocity of the impact in meters per second as defined in Table 1 and 2. In the experiments ${ }^{[46]}$, the maximum strain at this point was 322 microstrains, which corresponds to $V=3.5 \mathrm{~m} / \mathrm{s}$ in the derived formula. Note that this velocity is much smaller than the velocity obtained for the free fall of the shell from a given height. The strains 
at the four points on the shell as in Fig. 7, which were calculated by the Wagner model without a structural damping and with the reduced impact speed in ${ }^{[47]}$, were almost identical to those measured in experiments, even for the central angle of 30 degrees, in the time interval $0<\mathrm{t}<9 \mathrm{~ms}$. By using the developed MLM based model, the computational results with $3.5 \mathrm{~m} / \mathrm{s}$ initial impact velocity also show a very good match with experiment as shown in Fig. 8 (red and blue curves).

Moreover, for the first strain peak, the error of the prediction by the developed MLM model for $3.5 \mathrm{~m} / \mathrm{s}$ entry velocity is about $10 \%$, whereas the computed second strain peaks are almost identical to the experimental results. The error for $4.429 \mathrm{~m} / \mathrm{s}$ entry velocity case are about twice of the values for $3.5 \mathrm{~m} / \mathrm{s}$ entry velocity. The two-norm errors of strain (multiplied by $10^{6}$ ) between experiment and computed results are listed in Table 3.

Table 3 Two-norm errors of the cylindrical shell strain results

\begin{tabular}{|l|l|l|l|l|}
\hline Two-norm error $\left(1 \times 10^{6}\right)$ & 0 degree & 10 degree & 20 degree & 30 degree \\
\hline MLM, V $=4.429 \mathrm{~m} / \mathrm{s}$ & 70.5208 & 66.0776 & 52.7588 & 35.4429 \\
\hline MLM, V $=3.5 \mathrm{~m} / \mathrm{s}$ & 33.3712 & 36.9143 & 26.6271 & 19.2779 \\
\hline
\end{tabular}

The number of elastic modes in ${ }^{[47]}$ was larger than in the later studies of the same problem by other methods because the added mass matrix was calculated analytically in ${ }^{[47]}$. It was concluded in ${ }^{[47]}$ that models with non-extensible neutral surface of the shell may provide misleading results for the strains.

Sun ${ }^{[10]}$ simulated the same problem for the conditions of experiments ${ }^{[46]}$ by matching the Wagner and von Karman theories of water impact for the initial 0.0012s and BEM for the rest of the entry. As shown in Fig. 8, the Wagner + BEM model (dash dotted line) gives similar results as the present model, whilst the prediction by the von Karman + BEM model (dotted line) agrees better with the experimental results. This indicates that the impact force during the very short period of initial impact stage is vital for the accurate prediction of the dynamic response of structure. This also implies that the developed model could provide same level of accuracy compared with BEM, whilst the semi-analytical model of this paper is considerably more efficient.

The penetration velocity and acceleration of the cylinder predicted by different methods are shown in Fig. 9. The dotted line is for the corresponding rigid body case, where the hydrodynamic pressure is calculated by the same formulations as in Section 2.2 but without the terms related to elastic deformations. It is clear from the figure that the elastic deformation of the shell has a significant effect on the rigid-body motion of the cylinder. For the elastic cases, the dynamic features calculated by different methods are similar, and the developed model agrees better with the Wagner + BEM method compared with the von Karman + BEM method, which is consistent with the results in Fig. 8. 


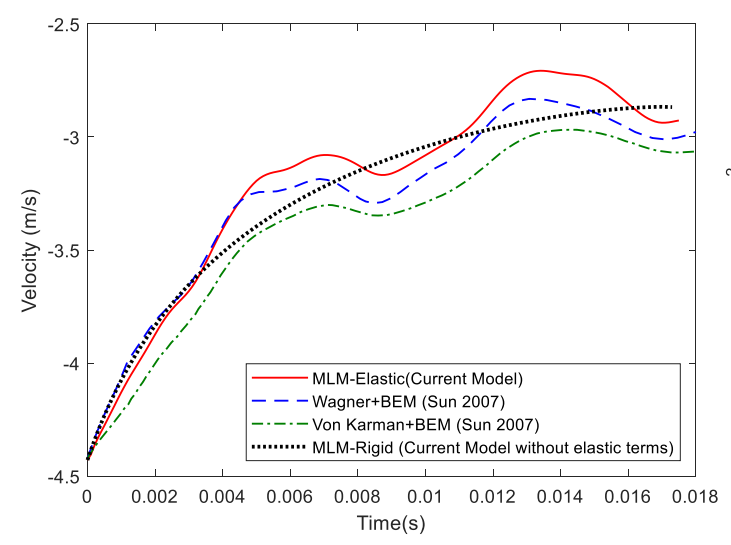

(a)

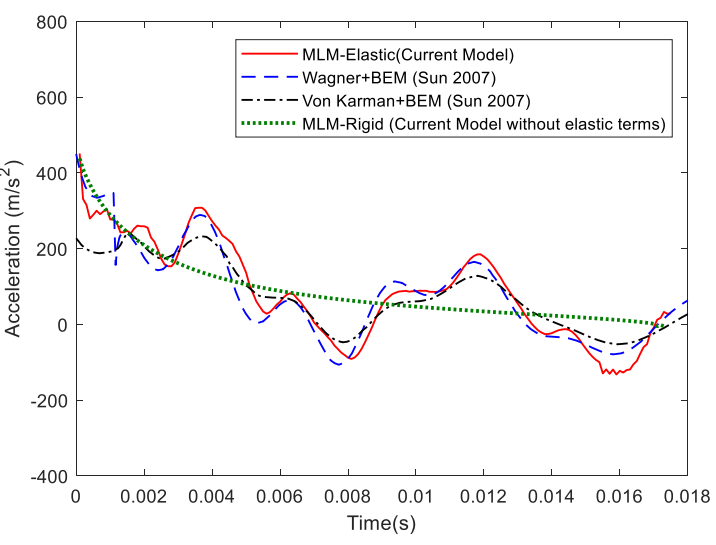

(b)

Fig. 9 The vertical velocity (a) and acceleration (b) of the cylinder with and without account for elastic deformations.

The effect of elastic mode number on the strains was investigated and the results are shown in Fig. 10. It is seen that three elastic modes provide reasonable results. This agrees with the results from ${ }^{[47]}$, where it was obtained that five elastic modes provide qualitatively correct strains, and the strain evolutions do not significantly for the number of modes greater than ten.

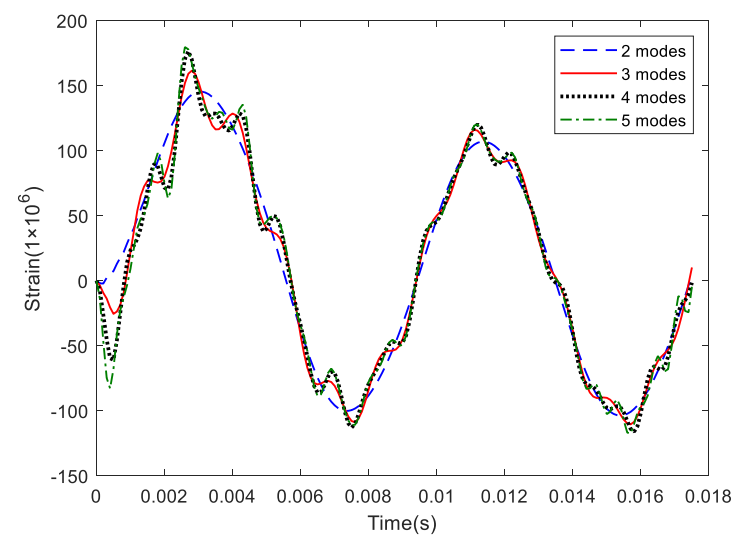

Fig. 10 The strain at 30 degree central angle of the cylindrical shell calculated with different number of elastic modes.

\section{Conclusion}

In this study, a hydroelastic slamming simulation framework consisting of analytical hydrodynamic computation and coupled rigid-elastic structural response calculation is established. It is designed to handle bodies with arbitrary symmetric shapes. For the hydrodynamic computation, the MLM model, which includes the non-linear term in the pressure Bernoulli equation, is used. The governing equations of structural dynamics account for interaction between elastic and rigid motion modes. The fluid and structure interaction is conducted in an iterative way (i.e. prediction and correction) until convergence. This portioned type of FSI coupling strategy, which is commonly used in many CFD based FSI models, provides good convergence performance in this study.

The accuracy of the developed model is validated through various cases, including wedge water entry with both constant velocity and free-drop conditions, as well as cylindrical shell freedrop onto water surface. The vertical forces, structural deflection and strains are compared with the available experimental and computational results from the literature. The comparison shows that the inclusion of the non-linear term of Bernoulli equation in MLM model has indeed provide better 
accuracy for the hydrodynamic computation. Moreover, for the computed cases in this paper, the developed semi-analytical model shows similar or better accuracy compared with much more time consuming approaches such as BEM or SPH. However, the current semi-analytical model cannot handle the large flow deformation at the deep penetration stage. Nevertheless, the developed model at least provides an efficient and effective tool for the initial slamming stage, which normally generate the largest slamming force and therefore vital for the marine structural integrity checking in the early design stage.

\section{Acknowledgment}

This work was partially funded by the National Natural Science Foundation of China (Grant Nos. 51809035, 51639003 and 51679037), the National Key Research and Development Program of China (Grant No.2019YFC0312400), the China Postdoctoral Science Foundation (Grant No. 2018M641693), the Fundamental Research Fund for the Central Universities (Grant Nos. DUT19RC(4)028 and DUT2017TB05).

\section{Reference:}

1. Tuitman, J.T., Hydro-elastic response of ship structures to slamming induced whipping. 2010, Delft University of Technology.

2. Abrate, S., "Hull Slamming". In: Applied Mechanics Reviews. 64(2013), 060803.

3. Shams, A., Zhao, S. and Porfiri, M., "Hydroelastic slamming of flexible wedges: Modeling and experiments from water entry to exit". In: Physics of Fluids. 29(2017), 037107.

4. Jalalisendi, M. and Porfiri, M., "Water Entry of Cylindrical Shells: Theory and Experiments". In: AIAA Journal. 56(2018), 4500-4514.

5. Liao, K., Hu, C. and Duan, W., "Two-dimensional numerical simulation of an elastic wedge water entry by a coupled FDM-FEM method". In: Journal of Marine Science and Application. 12(2013), 163-169.

6. Sriram, V. and Ma.Q.W., "Improved MLPG_R method for simulating 2D interaction between violent waves and elastic structures". In: Journal of Computational Physics. 231(2012), 76507670.

7. Sun, Z., Zhang, G.Y., Zong, Z., Djidjeli, K. and Xing, J.T., "Numerical analysis of violent hydroelastic problems based on a mixed MPS-mode superposition method". In: Ocean Engineering. 179(2019), 285-297.

8. Sun, Z., Djidjeli, K., Xing, J.T. and Fai, C., "Coupled MPS-modal superposition method for 2D nonlinear fluid-structure interaction problems with free surface". In: Journal of Fluids and Structures. 61(2016), 295-323.

9. Zhang, Y. and Wan, D., "MPS-FEM coupled method for sloshing flows in an elastic tank". In: Ocean Engineering, (2017).

10. Sun, H., A Boundary Element Method Applied to Strongly Nonlinear Wave-Body Interaction Problems. 2007, Norwegian University of Science and Technology.

11. Sun, H. and Faltinsen, O.M., "Water impact of horizontal circular cylinders and cylindrical shells". In: Applied Ocean Research. 28(2006), 299-311. 
12. Von Karman, T., The Impact on a Sea Plane Floats During Landing, in Technical note for National Advisory Committee for Aeronautics. 1929: Washington, USA.

13. Wagner, H., "Uber Stoss-und Gleitvorgange an der Oberflache von Flussigkeiten". In: Zeitschrift fur Angewandte Mathematik und Mechanik. 12(1932), 193-215.

14. Schmieden, C., "Der Aufschlag von Rotations-kiirpern auf eine Wasseroberftache". In: ZAMM. 33(1953), 147-151.

15. Scolan, Y.-M. and Korobkin, A.A., "Three-dimensional theory of water impact. Part 1. Inverse Wagner problem". In: Journal of Fluid Mechanics. 440(2001), 293-326.

16. Korobkin, A.A., "The entry of an elliptical paraboloid into a liquid at variable velocity". In: Journal of applied mathematics and mechanics. 66(2002), 39-48.

17. Faltinsen, O.M., "Hydroelastic slamming". In: Journal of Marine Science and Technology. 5(2000), 49-65.

18. Korobkin, A.A. and Khabakhpasheva., T.I., "Regular wave impact onto an elastic plate". In: Journal of Engineering Mathematics. 55(2006), 127-150.

19. Scolan, Y.-M., "Hydroelastic behaviour of a conical shell impacting on a quiescent-free surface of an incompressible liquid". In: Journal of Sound and Vibration 277(2004), 163-203.

20. Khabakhpasheva, T.I. and Korobkin, A.A., "Elastic wedge impact onto a liquid surface: Wagner's solution and approximate models". In: Journal of Fluids and Structures. 36(2013), 32-49.

21. Shams, A. and Porfiri, M., "Treatment of hydroelastic impact of flexible wedges". In: Journal of Fluids and Structures. 57(2015), 229-246.

22. Korobkin, A., Guéret, R. and Malenica, Š., "Hydroelastic coupling of beam finite element model with Wagner theory of water impact". In: Journal of Fluids and Structures. 22(2006), 493-504.

23. Korobkin, A., "Analytical models of water impact". In: European Journal of Applied Mathematics. 15(2004), 821-838.

24. Zhao, R., Faltinsen, O.M. and Aarsnes, J., Water entry of arbitrary two-dimensional sections with and without flow separation, in 21st symposium on naval hydrodynamics. 1996, National Academy Press: Trondheim, Norway.

25. Logvinovich, G.V., Hydrodynamics of Flows with Free Boundaries. 1969: Naukova Dumka.

26. Korobkin, A.A. Three-dimensional nonlinear theory of water impact. in 18th International Congress of Mechanical Engineering. 2005.

27. Wraith, R., Pressure loads on ship hull plating caused by slamming. 1998, University of Melbourne.

28. Tassin, A., Piro, D.J., Korobkin, A.A., Maki, K.J. and Cooker, M.J., "Two-dimensional water entry and exit of a body whose shape varies in time". In: Journal of Fluids and Structures. 40(2013), 317-336.

29. Küttler, U. and Wall, W.A., "Fixed-point fluid-structure interaction solvers with dynamic relaxation". In: Computational Mechanics. 43(2008), 61-72.

30. Megson, T.H.G., Structural and Stress Analysis. 2020: Butterworth-Heinemann.

31. Rao, S.S., Mechanical Vibrations. fifth ed. 2011: Pearson Education, Inc.

32. Newmark, N.M., "A Method of Computation for Structural Dynamics". In: Journal of the Engineering Mechanics Division. 85(1959), 67-94.

33. In: (!!! INVALID CITATION !!! \{\}).

34. Khabakhpasheva, T., A.Korobkin, Maki, K. and Seng, S., Water entry and exit with large displacements by simplified models, in 31st International Workshop on Water Waves and 
Floating Bodies, R.Beck and K.J. Maki, Editors. 2016: Plymouth, Michigan, USA.

35. Arfken, G.B., Weber, H.J. and Harris, F.E., Mathematical Methods for Physicists-A Comprehensive Guide. 2012: Academic Press.

36. Lakshmynarayanana, P.A. and Temarel, P., "Application of CFD and FEA coupling to predict dynamic behaviour of a flexible barge in regular head waves". In: Marine Structures. 65(2019), 308-325.

37. Mcvicar, J., Lavroff, J., Davis, M.R. and Thomas, G., "Fluid-structure interaction simulation of slam-induced bending in large high-speed wave-piercing catamarans". In: Journal of Fluids and Structures. 82(2018), 35-58.

38. Irons, B. and Tuck, R.C., "A version of the Aitken accelerator for computer implementation". In: International Journal for Numerical Methods in Engineering. 1(1969), 275-277.

39. Piro, D.J. and Maki, K.J., "Hydroelastic analysis of bodies that enter and exit water". In: Journal of Fluids and Structures. 37(2013), 134-150.

40. Korobkin, A.A. and Khabakhpasheva, T.I., Impact Of Elastic Body On The Deep And Shallow Water, in 32nd International Conference on Ocean, Offshore and Arctic Engineering (OMAE). 2013: Nantes, France. p. 1-10.

41. Tassin, A., Korobkin, A.A. and Cooker, M.J., "On analytical models of vertical water entry of a symmetric body with separation and cavity initiation". In: Applied Ocean Research. 48(2014), 33-41.

42. Ferrand, M., Laurence, D.R., Rogers, B.D., Violeau, D. and Kassiotis, C., "Unified semi-analytical wall boundary conditions for inviscid, laminar or turbulent flows in the meshless SPH method". In: International Journal for Numerical Methods in Fluids. 71(2013), 446-472.

43. Panciroli, R. and Porfiri, M., "Analysis of hydroelastic slamming through particle image velocimetry". In: Journal of Sound and Vibration. 347(2015), 63-78.

44. Panciroli, R., Abrate, S., Minak, G. and Zucchelli, A., "Hydroelasticity in water-entry problems: Comparison between experimental and SPH results". In: Composite Structures. 94(2012), 532539.

45. Faltinsen, O.M., "Water entry of a wedge by hydroelastic orthotropic plate theory". In: Journal of Ship Research. 43(1999), 180-193.

46. Shibue, T., Ito, A. and Nakayama, E., "Structural resposne analysis of cylinders under water impact". In: Journal of the Society of Naval Architects of Japan. 174(1993).

47. Ionina, M.F. and Korobkin, A.A., Water impact on cylindrical shells, in International Workshop on Water Waves and Floating Bodies. 1999: Port Huron, MI, USA.

48. Ionina, M.F., "Penetration of an elastic circular cylindrical shell into an incompressible liquid". In: Journal of applied mechanics and technical physics. 40(1999), 1163-1172. 